\title{
Article
}

\section{The Baryonic Collapse Efficiency of Galaxy Groups in the RESOLVE and ECO Surveys}

Eckert, Kathleen, D, Kannappan, Sheila, J, Lagos, Claudio del P., Baker, Ashley, D, Berlind, Andreas, A, Stark, David, V, Moffett, Amanda, J, Nasipak, Zachary and Norris, Mark, A

Available at https://clok.uclan.ac.uk/20099/

Eckert, Kathleen, D, Kannappan, Sheila, J, Lagos, Claudio del P., Baker, Ashley, D, Berlind, Andreas, A, Stark, David, V, Moffett, Amanda, J, Nasipak, Zachary and Norris, Mark, A orcid iconORCID: 0000-0002-7001-805X (2017) The Baryonic Collapse Efficiency of Galaxy Groups in the RESOLVE and ECO Surveys. The Astrophysical Journal, 849 (1). ISSN 0004-637X

It is advisable to refer to the publisher's version if you intend to cite from the work. http://dx.doi.org/10.3847/1538-4357/aa8e97

For more information about UCLan's research in this area go to

http://www.uclan.ac.uk/researchgroups/ and search for <name of research Group>.

For information about Research generally at UCLan please go to http://www.uclan.ac.uk/research/

All outputs in CLoK are protected by Intellectual Property Rights law, including Copyright law. Copyright, IPR and Moral Rights for the works on this site are retained by the individual authors and/or other copyright owners. Terms and conditions for use of this material are defined in the policies page.

\section{CLoK}

Central Lancashire online Knowledge www.clok.uclan.ac.uk

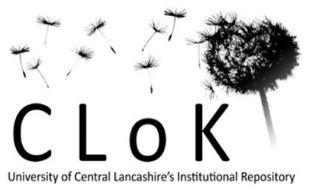




\title{
The Baryonic Collapse Efficiency of Galaxy Groups in the RESOLVE and ECO Surveys
}

\author{
Kathleen D. Eckert ${ }^{1,2}$ (1), Sheila J. Kannappan ${ }^{1}$, Claudia del P. Lagos ${ }^{3}$, Ashley D. Baker ${ }^{1,2}$, Andreas A. Berlind ${ }^{4}$ (i), \\ David V. Stark ${ }^{5}$ (D), Amanda J. Moffett ${ }^{4}$, Zachary Nasipak ${ }^{1}$, and Mark A. Norris ${ }^{6}$ \\ ${ }^{1}$ Department of Physics and Astronomy, University of North Carolina, 141 Chapman Hall CB 3255, Chapel Hill, NC 27599, USA; keckert@physics.unc.edu \\ ${ }^{2}$ Department of Physics and Astronomy, University of Pennsylvania, 209 South 33rd Street, Philadelphia, PA 19104-6396, USA \\ ${ }^{3}$ International Centre for Radio Astronomy Research (ICRAR), The University of Western Australia, 35 Stirling Highway, Crawley, WA 6009, Australia \\ ${ }^{4}$ Department of Physics and Astronomy, Vanderbilt University, PMB 401807, 2401 Vanderbilt Place, Nashville, TN 37240-1807, USA \\ ${ }^{5}$ Kavli IPMU (WPI), UTIAS, The University of Tokyo, Kashiwa, Chiba, 277-8583, Japan \\ 6 Jeremiah Horrocks Institute, University of Central Lancashire, Preston, PR1 2HE, UK \\ Received 2017 April 21; revised 2017 September 6; accepted 2017 September 20; published 2017 October 26
}

\begin{abstract}
We examine the $z=0$ group-integrated stellar and cold baryonic (stars + cold atomic gas) mass functions (group SMF and CBMF) and the baryonic collapse efficiency (group cold baryonic to dark matter halo mass ratio) using the RESOLVE and ECO survey galaxy group catalogs and a GALFORM semi-analytic model (SAM) mock catalog. The group SMF and CBMF fall off more steeply at high masses and rise with a shallower low-mass slope than the theoretical halo mass function (HMF). The transition occurs at the group-integrated cold baryonic mass $M_{\text {bary }}^{\text {cold }} \sim 10^{11} M_{\odot}$. The SAM, however, has significantly fewer groups at the transition mass $\sim 10^{11} M_{\odot}$ and a steeper low-mass slope than the data, suggesting that feedback is too weak in low-mass halos and conversely too strong near the transition mass. Using literature prescriptions to include hot halo gas and potential unobservable galaxy gas produces a group BMF with a slope similar to the HMF even below the transition mass. Its normalization is lower by a factor of $\sim 2$, in agreement with estimates of warm-hot gas making up the remaining difference. We compute baryonic collapse efficiency with the halo mass calculated two ways, via halo abundance matching (HAM) and via dynamics (extended all the way to three-galaxy groups using stacking). Using HAM, we find that baryonic collapse efficiencies reach a flat maximum for groups across the halo mass range of $M_{\text {halo }} \sim 10^{11.4-12} M_{\odot}$, which we label "nascent groups." Using dynamics, however, we find greater scatter in baryonic collapse efficiencies, likely indicating variation in group hot-to-cold baryon ratios. Similarly, we see higher scatter in baryonic collapse efficiencies in the SAM when using its true groups and their group halo masses as opposed to friends-of-friends groups and HAM masses.
\end{abstract}

Key words: galaxies: halos - galaxies: luminosity function, mass function - surveys

Supporting material: machine-readable table

\section{Introduction}

Galaxies form and evolve within the context of their local environment, which can be characterized by group dark matter halos. At $z=0$, galaxies in low-mass halos tend to be star forming with abundant cold gas, while in large groups and clusters, the galaxy population is quenched of star formation with little cold gas (e.g., Davies \& Lewis 1973; Kennicutt 1983a; Haynes et al. 1984). In the largest clusters, the dominant baryonic component is the hot X-ray-emitting gas (e.g., Mitchell et al. 1977; Giodini et al. 2009), while in lower-mass halos, the halo gas temperatures are too low to emit X-rays, presumably leaving the majority of the gas in an unobservable warm-hot state (i.e., the warm-hot intergalactic medium, WHIM; Cen \& Ostriker 2006). The collapsed baryons (in the form of stars and cold gas) dominate the observable baryonic component of such low-mass groups.

Previous works examining the baryonic content of clusters have used X-ray data to study the hot gas, finding that the halo gas dominates the baryonic content for group halos with masses $>10^{13-13.5} M_{\odot}$ and that even in the highest-mass clusters probed $\left(\sim 10^{15} M_{\odot}\right)$, the universal baryon fraction is not reached (e.g., Ramella et al. 2004; Gonzalez et al. 2007; Giodini et al. 2009; Balogh et al. 2011). These works use cluster member dynamics or X-ray luminosity calibrations to measure halo masses. For lower-mass groups for which X-rays are difficult to detect and galaxy dynamics harder to measure (due to few members), studies have used halo abundance matching (HAM) or the halo occupation distribution (HOD) method to study the stellar content of groups (Moster et al. 2010; Leauthaud et al. 2012a; Behroozi et al. 2013). These studies find that the group stellar fraction (group stellar mass divided by group halo mass) peaks at halo masses $\sim 10^{12}$ $M_{\odot}$ and decreases toward higher and lower halo masses.

These previous studies have focused on the stellar content of groups, leaving out the contribution from cold gas (the reservoir for future star formation), which can dominate the galaxy mass for galaxies with cold baryonic mass $M_{\text {bary }}^{\text {cold }}<10^{9.9} M_{\odot}$, the gas richness threshold mass defined in Kannappan et al. (2013), hereafter K13. Even at higher galaxy masses, star-forming galaxies have $\mathrm{HI}$ gas-to-stellar mass ratios typically ranging from 0.1 to 1 (e.g., Catinella et al. 2013; Kannappan et al. 2013; Brown et al. 2015). In this work, we define the term "cold baryonic mass" to mean the mass in stars and cold atomic gas (see Section 2.3.1), neglecting other cold gas components. In a previous work, we showed that the low-mass slope of the galaxy cold baryonic mass function rises more steeply than that of the stellar mass function (Eckert et al. 2016, hereafter E16). We also found complex structure after breaking the baryonic mass function into different group halo mass regimes. In the intermediate group halo mass regime $\sim 10^{11.4}-10^{12} M_{\odot}$, we found a flat low-mass slope, potentially a signature of group formation processes such as stripping and merging. We refer to 
groups in this mass range as "nascent groups," where galaxies first start to come together to form larger structures.

These results motivate our desire to study group-integrated mass functions and the group baryonic collapse efficiency (the cold baryonic group mass divided by the group halo mass). To perform this study, we use two volume-limited surveys with groups ranging in halo mass from $\sim 10^{11} M_{\odot}$ to $10^{14.5} M_{\odot}$. The smaller, RESOLVE-B, is complete to an individual galaxy cold baryonic mass limit of $M_{\text {bary }}^{\text {cold }} \sim 10^{9.1} M_{\odot}$. The larger, ECO, encompasses the RESOLVE-A subvolume and is complete to galaxy $M_{\text {bary }}^{\text {cold }} \sim 10^{9.4} M_{\odot}$. We also construct a mock catalog from the GALFORM semi-analytic model (SAM) of GonzalezPerez et al. (2014) to compare with the data.

In Section 2, we describe the data and methods used in this work to measure the mass of groups in terms of stellar, cold baryonic, and group halo mass. In Section 3, we analyze the group-integrated stellar and cold baryonic mass functions (SMF and CBMF) and examine the stellar and cold baryonic fractions of groups, finding a broad peak in baryonic collapse efficiency from $10^{11.4}-10^{12} M_{\odot}$ across the nascent group regime. In Section 4, we discuss the implications of our results on nascent group formation and undetected forms of gas. Finally, in Section 5, we summarize our conclusions.

\section{Data and Methods}

Below we present a brief overview of the two data sets used in this work, including their relative merits. We also present a description of the data used to construct the group-integrated properties for the two data sets. Finally, we describe the mock catalog created from the GALFORM SAM of Gonzalez-Perez et al. (2014).

\subsection{Data Sets}

In this work, we use two data sets, the REsolved Spectroscopy of a Local VolumE survey (RESOLVE; S. J. Kannappan et al. 2017, in preparation) and the Environmental COntext catalog (ECO; Moffett et al. 2015, hereafter M15). Both data sets are volume limited and have been constructed using the SDSS main redshift survey (Strauss et al. 2002), filling in incompleteness due to fiber collisions and pipeline photometry issues (see Blanton et al. 2005a) with data from several other redshift surveys as described in E16. For both surveys, we define membership based on group redshift using a buffer region to recover galaxies whose peculiar velocities place them outside the survey limits (see Section 2.3). Although the RESOLVE survey has greater completeness and deeper photometric and $\mathrm{H}$ I data, the ECO catalog covers a much larger volume, providing better statistics and a wider range of group halo masses.

The RESOLVE survey covers a $>50,000 \mathrm{Mpc}^{3}$ volume over two equatorial strips ranging in redshift from 4500 to $7000 \mathrm{~km} \mathrm{~s}^{-1}$ (see Eckert et al. 2015, hereafter E15, for more details). The $\sim 13,700 \mathrm{Mpc}^{3}$ RESOLVE-B footprint coincides with SDSS Stripe 82, while the larger RESOLVE-A footprint is surrounded by the ECO catalog. RESOLVE-B has extra redshift completeness due to repeated observations by the SDSS (see E16). Due to the extra redshift completeness, we dropped the RESOLVE-B luminosity completeness limit to $M_{r, \text { tot }}=-17.0$, below the nominal luminosity completeness limit of $M_{r \text {,tot }}=-17.33$, which corresponds to the SDSS apparent magnitude survey limit of 17.77 at the outer redshift boundary using the RESOLVE total magnitudes from Eckert et al. (2015). The RESOLVE-B volume contains 486 galaxies brighter than this limit and 344 groups, 286 of which have $N=1$ member.

RESOLVE-B is covered by deep ugriz coadds in the SDSS (Aihara et al. 2011), as well as shallow JHK 2MASS (Skrutskie et al. 2006) and deep $Y H K$ UKIDSS data (Hambly et al. 2008). In addition, it has nearly complete coverage by the GALEX MIS depth survey $(\sim 1500 \mathrm{~s})$ in the NUV (Morrissey et al. 2007), plus Swift uvm2 imaging for 19 galaxies (E15). The RESOLVE H I survey, presented in Stark et al. (2016), provides unconfused (or deconfused) H I detections or strong upper limits $\left(1.4 M_{\mathrm{H} \mathrm{I}}<0.05 M_{\text {star }}\right)$ for $87 \%$ of galaxies brighter than $M_{r \text {,tot }}-17.0$ or having estimated $M_{\text {bary }}^{\text {cold }}>10^{9.0} M_{\odot}$, based on calibrations of the relationship between galaxy gas-tostellar mass ratio and color (the photometric gas fractions technique described in E15).

The ECO catalog covers a volume of $\sim 442,700 \mathrm{Mpc}^{3}$, which is $\sim 32$ times larger than RESOLVE-B and encompasses RESOLVE-A. Although less complete in terms of redshift coverage, the ECO volume provides statistical power that the smaller RESOLVE-B subvolume cannot provide, having 9443 galaxies brighter than the luminosity limit of -17.33 and 6746 groups, of which 5723 are groups of $N=1$.

Although ECO has uniform shallow coverage over ugrizJHK from SDSS and 2MASS, deeper imaging from UKIDSS is limited to the RESOLVE-A region and MIS depth NUV from GALEX covers $\sim 45 \%$ of ECO (including most of RESOLVEA). Fractional-mass limited $\mathrm{HI}$ data are available for the RESOLVE-A subvolume within ECO, providing a similar quality of data to RESOLVE-B. Additional coverage is provided by the flux-limited $21 \mathrm{~cm}$ ALFALFA survey's $\alpha 40$ catalog (Haynes et al. 2011), which yields H I detections for galaxies with $M_{\mathrm{H}} \gtrsim 10^{9} M_{\odot}$ at ECO redshifts. We computed upper limits for galaxies with ALFALFA non-detections, but $\sim 84 \%$ of those are weak (i.e., $1.4 M_{\mathrm{HI}}^{\text {limit }}>0.05 M_{\text {star }}$ ). For ECO galaxies without $\mathrm{HI}$ data or having only a weak upper limit, we rely on gas mass estimates using the photometric gas fractions technique described in E15, which provides full probability distributions for the gas mass, not just point estimates as in previous works (e.g., Kannappan 2004; Li et al. 2012; Catinella et al. 2013).

In E16, we computed the galaxy stellar and baryonic mass completeness limits for the RESOLVE-B and ECO volumes by examining the stellar and baryonic mass-to-light ratio distributions near each survey's respective luminosity completeness limit. For RESOLVE-B, we find that the stellar and baryonic mass completeness limits are $M_{\text {star }}=10^{8.7} M_{\odot}$ and $M_{\text {bary }}^{\text {cold }}=10^{9.1} M_{\odot}$. For ECO, they are $M_{\text {star }}=10^{8.9} M_{\odot}$ and $M_{\text {bary }}^{\text {cold }}=10^{9.4} M_{\odot}$.

To determine our group mass completeness limits, we note that at low group mass, we are dominated by $N=1$ groups, so these galaxy mass completeness limits should roughly translate to group-integrated mass limits. There may, however, be groups consisting entirely of galaxies below our luminosity completeness limit (such as dwarf associations; Tully et al. 2006). To quantify how many such groups we may be missing, we examine the number of groups in RESOLVE-B with group-integrated stellar or cold baryonic mass greater than the shallower ECO mass completeness limits, but having no galaxy brighter than the ECO luminosity limit $(-17.33)$. We find that $<1 \%$ of RESOLVE-B groups fit this criteria, implying that our galaxy completeness limits are sufficient. 
Due to the superior spectroscopic completeness of RESOLVE-B relative to SDSS (E16), we can consider RESOLVE-B to be a truly complete data set. For ECO, however, we know that we are missing galaxies due to both fiber collisions and surface brightness incompleteness, despite efforts to account for galaxies through merging of several spectroscopic surveys (M15, E16). To address this incompleteness in ECO, E16 computed galaxy completeness corrections as a function of luminosity and color by comparing the completeness of RESOLVE-B and ECO relative to the main SDSS redshift survey over luminosity-color space (see M15 and E16 for details). These completeness corrections have been applied as weights in the galaxy mass functions in E16. We describe how we translate the weights to group completeness corrections in Section 2.4.

\subsection{Photometry and Galaxy Stellar and Cold Baryonic Masses}

In this work, we use reprocessed photometry, as described in E15 and M15 for the RESOLVE and ECO data sets, respectively. Our reprocessing addresses several issues in the catalog photometry. For the SDSS data, we use the improved sky background subtraction of Blanton et al. (2011), and for the IR data, we perform additional custom background subtraction. By enforcing the same elliptical apertures (based on the high $\mathrm{S} / \mathrm{N}$ gri coadded image) across all bands, we are able to measure total galaxy magnitudes in all bands using three nonparametric methods, the comparison of which yields systematic error estimates. Our methods allow for color gradients in galaxies as opposed to the algorithms used for the SDSS catalog photometry, which suppress color gradients (Stoughton et al. 2002).

These improvements yield brighter magnitudes, larger radii, overall bluer colors, and more real scatter in color (see Figures 3 and 4 of E15). These last two points imply that galaxy star formation rates are higher and star formation histories are more varied than previously reported. In K13, which used similarly processed photometry for the Nearby Field Galaxy Survey (NFGS, Jansen \& Kannappan 2001), low-mass gas-rich galaxies, traditionally regarded as poor star formers, were found to be doubling their masses over the last gigayear (Gyr).

RESOLVE and ECO stellar masses (E15, M15) were computed using the Bayesian spectral energy distribution (SED) fitting approach described in K13 (see also Kannappan $\&$ Gawiser 2007). The code produces a likelihood-weighted mass distribution for each galaxy based on the full model grid considered. Briefly, the grid consists of an old and young stellar population, each populated with a Chabrier IMF. The old stellar population is modeled as a burst with age ranging from 2 to 12 Gyr. The young stellar population is modeled either as continuous star formation starting $1015 \mathrm{Myr}$ ago and continuing to a turnoff sometime in the last $0-195 \mathrm{Myr}$ or as a single quenching burst with age ranging from 360 to $1015 \mathrm{Myr}$. The young stellar population can contribute from $0.1 \%$ to $94.1 \%$ of the stellar mass. The grid includes four metallicities ranging from $Z=0.004-0.05$, and 11 optical depth dust values (ranging from $\tau_{v}=0-1.2$ ) are applied to the young stellar population using the dust law from Calzetti (2001). We generally use the full mass likelihood distribution in this work, but when we assign a single value for the galaxy's stellar mass, we take the median of the likelihood-weighted stellar mass distribution.

As previously mentioned, cold baryonic mass in this work is defined as the stellar plus the cold atomic gas mass. Generally, the atomic gas dominates the cold gas mass of galaxies, although large spirals may have significant reservoirs of molecular gas. The total gas mass in large spirals, however, is typically $\lesssim$ half of the stellar mass (Casoli et al. 1998; Kannappan et al. 2013; Boselli et al. 2014). RESOLVE-B and ECO both have H I data available with varying depth and coverage. Although RESOLVE's coverage is fractional-mass limited and nearly complete, ECO has fractional-mass limited data only in the RESOLVE-A subvolume and relies on the flux-limited ALFALFA survey elsewhere, which provides mostly weak upper limits. In this work, we define the atomic gas mass to be $1.4 M_{\mathrm{H} \text { I }}$ to account for the contribution from helium.

To supplement the H I data, we use the photometric gas fraction (PGF) technique to estimate gas-to-stellar mass $(\mathrm{G} / \mathrm{S})$ ratios as described in E15. The estimators are based on a model fit to the $2 \mathrm{D}$ distribution of $\log (\mathrm{G} / \mathrm{S}$ ) versus color (or "modified color," a linear combination of color and axial ratio) to produce $\log (\mathrm{G} / \mathrm{S})$ distributions for each galaxy. These estimates of $\log (\mathrm{G} / \mathrm{S})$ are created using the RESOLVE-A data set and are therefore ideal for use on volume-limited surveys, as validated by testing on the RESOLVE-B H I data set in E15.

To compute cold baryonic mass, we perform a "pseudoconvolution" of the stellar mass likelihood distribution for a given galaxy with the $\mathrm{H}$ I mass likelihood distribution implied by its $\mathrm{H}$ I data (for good detections) or inferred from its PGFestimated $\log (\mathrm{G} / \mathrm{S}$ ) distribution (for missing, low $\mathrm{S} / \mathrm{N}$, or badly confused detections). The details are provided in E16. This algorithm results in a cold baryonic mass likelihood distribution, from which we can take the median if a single value for the galaxy's baryonic is necessary. (We use the full distribution by default.)

\subsection{Group Stellar, Cold Baryonic, and Halo Masses}

The fact that RESOLVE and ECO are volume limited enables optimal group finding, for which we use the Friends-ofFriends (FOF) algorithm from Berlind et al. (2006). This algorithm links galaxies that are within specified projected and line-of-sight linking lengths into groups. The projected and line-of-sight linking lengths determined in Berlind et al. (2006), respectively, $b_{\perp}=0.14$ and $b_{\|}=0.75$ times the mean separation between objects, were designed to reproduce the multiplicity function and projected sizes of groups with $N>10$ members. Based on our own work as well as those of Duarte \& Mamon (2014) and Robotham et al. (2011), we use projected and line-of-sight linking lengths better geared toward the recovery of low- $N$ groups and dynamical masses: $b_{\perp}=0.07$ and $b_{\|}=1.1$ times the mean separation between objects (for more details, see Section 3.5.1 of E16). Since the RESOLVE-B subvolume is small (and overdense due to cosmic variance; E16), we fix its linking lengths to equal those computed for a version of ECO that extends to $M_{r \text {,tot }}=-17.0$, i.e., a version of ECO with depth analogous to RESOLVE-B but without its overdensity (see M15). After running the FOF code, each galaxy is assigned a group. We consider galaxies that are identified as being alone in their halo as $N=1$ groups with isolated "central" galaxies.

As a consequence of the FOF group-finding algorithm, many isolated $N=1$ groups are falsely linked into pairs. To cut down the number of false pairs, we use a mock catalog, for which we know the true pairs, to identify a region in $\Delta c z-R_{\text {proj }}$ space containing $95 \%$ of true pairs. Breaking up all pairs 
Table 1

RESOLVE and ECO Group Catalog Description

\begin{tabular}{|c|c|}
\hline Column & Description \\
\hline 1 & RESOLVE or ECO galaxy ID \\
\hline 2 & Group ID \\
\hline 3 & Group $N$ \\
\hline 4 & Group R.A. \\
\hline 5 & Group decl. \\
\hline 6 & Group cz \\
\hline 7 & HAM halo mass $\left(M_{\text {halo }}^{\text {HAM }}\right.$, based on group $\left.L_{r}\right)$ \\
\hline 8 & HAM halo mass $\left(M_{\text {halo }}^{\mathrm{HAM}}\right.$, based on group $\left.M_{\text {star }}\right)$ \\
\hline 9 & Dynamical halo mass $\left(\hat{M}_{\text {halo }}^{\text {dyn }}\right.$, scaled by $\left.\mathrm{A}=9.9\right)$ \\
\hline 10 & Stacked dynamical halo mass (scaled by $\mathrm{A}=9.9$ ) \\
\hline 11 & Hybrid dynamical halo mass $\left(M_{\text {halo }}^{\mathrm{H} \text {-dyn }}\right)$ \\
\hline 12 & Group-integrated stellar mass (group $M_{\text {star}}$ ) \\
\hline 13 & Group-integrated cold baryonic mass (group $M_{\text {bary }}^{\text {cold }}$ ) \\
\hline 14 & Central galaxy flag (brightest galaxy in $L_{r}$ ) \\
\hline 15 & Group velocity dispersion ( $\sigma_{\text {grp }}$, using the Gapper method) \\
\hline 16 & Group projected radius ( $R_{\text {proj }}$, using percentile method) \\
\hline
\end{tabular}

(This table is available in its entirety in machine-readable form.)

outside this region into groups of $N=1$, the percentage of true pairs in the FOF group catalog increases from $62 \%$ to $73 \%$. For further information on the algorithm used to break up false pairs, see the Appendix.

To describe the mass content of groups in this work, we use three different types of metrics: the group-integrated stellar and cold baryonic mass (group $M_{\text {star }}$ and $M_{\text {bary }}^{\text {cold }}$ ), the group halo mass determined through halo abundance matching $\left(M_{\text {halo }}^{\mathrm{HAM}}\right)$, and the group total mass determined from dynamics $\left(M_{\mathrm{halo}}^{\mathrm{dyn}}\right)$. We have also provided machine-readable tables with the group information and quantities described in the following sections for the RESOLVE and ECO galaxy catalogs. The columns of the data provided in the two tables ${ }^{7}$ are given in Table 1.

\subsubsection{Group-integrated Stellar and Cold Baryonic Masses}

The group-integrated stellar and cold baryonic masses (group $M_{\text {star }}$ and $M_{\text {bary }}^{\text {cold }}$ ) are the respective sums of the stellar and cold baryonic masses of all galaxies within the group. To compute the likelihood distributions of these integrated masses for each group, we use a pseudo-convolution method similar to the method used to compute the galaxy baryonic mass in E16. We do this so that we can use the mass likelihood distributions for each group with the cross-bin sampling technique of E16 to determine smooth group-integrated mass functions and uncertainty bands (see Section 3.1).

Briefly, for groups with $N=1$ member, the group-integrated mass likelihood distribution is the mass likelihood distribution of the single galaxy. For groups with $N>1$ members, we start with the two least massive galaxies. First, we compute the mass likelihood distributions of both galaxies divided into bins of linear spacing $\Delta M$, which can range from $1 / 100$ to $1 / 2$ of the smallest mass with likelihood $>1 \mathrm{e}^{-4}$. The range accounts for

\footnotetext{
7 The coordinates provided in the table have been updated to reflect the changes described in the erratum to E15 that affect 29 galaxies in RESOLVEB. These updated coordinates change the measured $R_{\text {proj }}$ for the affected groups by less than $2 \%$. Group stellar masses have not been updated to reflect the changes described in the erratum as most differences in the stellar mass estimates are $<0.03$ dex.
}

the potentially large difference in mass between the two galaxies (possibly a factor of 10-100) to keep the calculation from taking too long. We then perform the "pseudo-convolution" by computing the new mass and likelihood for each possible mass combination of the two galaxies. This pseudoconvolution is repeated with each resulting mass likelihood distribution and successively more massive galaxy in the group (updating $\Delta M$ for each round) to produce the group mass likelihood distribution. To assign a specific stellar or baryonic mass value to a group, we use the median of its mass likelihood distribution. The typical uncertainties on these group-integrated masses are comparable to the factor of $\sim 1.5-2$ uncertainties on stellar masses from SED fitting.

We can estimate the neglected stellar and cold baryonic mass contributions from satellites below the survey limit floor by using the satellite mass functions from E16. First, we normalize the satellite mass functions in each group halo mass regime presented in E16 (divisions at $10^{11.4} M_{\odot}, 10^{12} M_{\odot}$, and $\left.10^{13.5} M_{\odot}\right)$. Then, we fit a line to the low-mass slope to extrapolate the satellite mass function below our survey mass limits, and we integrate the extrapolated total mass in satellites from our survey limit down to $M_{\text {star }}$ or $M_{\text {bary }}^{\text {cold }}=10^{6} M_{\odot}$. In the nascent group halo mass regime $\left(M_{\text {halo }}=10^{11.4-12} M_{\odot}\right)$, we find that satellites below our mass limits contribute an $\sim 2 \%-4 \%$ to group $M_{\text {star }}$ and $\sim 7 \%-8 \%$ to group $M_{\text {bary }}^{\text {cold }}$. In the large-group halo mass and cluster mass regimes $\left(M_{\text {halo }}=\right.$ $10^{12.0}-10^{13.5} M_{\odot}$ and $\left.M_{\text {halo }}>10^{13.5} M_{\odot}\right)$, the contribution falls to $\sim 1.5 \%$ for group $M_{\text {star }}$ and $\sim 3 \%-4 \%$ for group $M_{\text {bary }}^{\text {cold }}$. To estimate the contribution from satellites below our luminosity limit for our lowest group halo mass regime (for which the satellite mass function is mostly incomplete), we scale the extrapolated slopes for the nascent and large-group halo satellite mass functions to estimate a range. In this halo mass regime, the satellite contribution to group $M_{\text {star }}$ ranges from $3 \%$ to $8 \%$ and that to group $M_{\text {bary }}^{\text {cold }}$ ranges from $8 \%$ to $14 \%$, depending on the slope used.

Although these contributions from galaxies below our survey floor have not been added to our group $M_{\text {star }}$ and group $M_{\text {bary }}^{\text {cold }}$, we do include mass from missing galaxies above our mass limit in ECO using the group completeness corrections described in Section 2.4.

\subsubsection{Group Halo Abundance Matching}

Group halo abundance matching (HAM) uses the cumulative number density of groups based on some group quantity (such as group luminosity) and matches the groups to halos of corresponding cumulative number density in simulations. In this work, we perform HAM using the halo mass function (HMF) from Warren et al. (2006), adopting a cosmological model with $H_{0}=70 \mathrm{~km} \mathrm{~s}^{-1} \mathrm{Mpc}^{-1}, \Omega_{m}=0.3$, and $\sigma_{8}=0.9$. We use both the group-integrated $r$-band luminosity (group $L_{r}$ ) down to the survey absolute magnitude floor and group $M_{\text {star }}$ to perform the matching. In particular, we note that the galaxy $L_{r}$ correlates more tightly with cold baryonic mass than with stellar mass (K13), suggesting that group $L_{r}$ should also correlate tightly with group $M_{\text {bary }}^{\text {cold }}$. Warren et al. (2006) use the standard simulation linking length parameter $b=0.2$, finding that these FOF halos are roughly equivalent to halos defined at $M_{280 b}$ or at an overdensity of 280 times the background density of matter. This method assumes that the group halo mass can be determined based on the group properties corresponding to the stellar or cold baryonic 


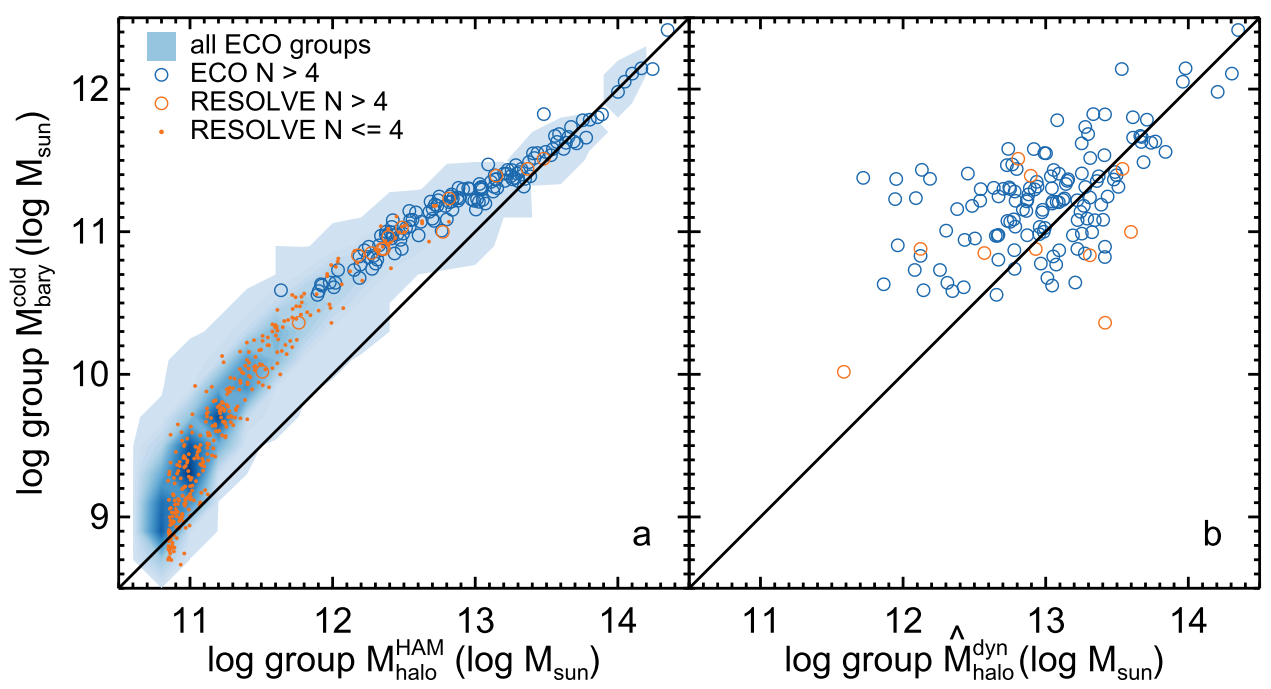

Figure 1. Group $M_{\text {bary }}^{\text {cold }}$ (including satellite completeness corrections; see Section 2.4) vs. $M_{\text {halo }}$ measured using (a) HAM and (b) dynamical estimates. ECO is shown in blue and RESOLVE is shown in orange. The black line shows the one-to-one relationship shifted down by 2 dex. Groups with $N>4$ are shown as open circles. $M_{\text {halo }}^{\text {HAM }}$ is closely correlated to the group $M_{\text {bary }}^{\text {cold }}$ since we used the group $r$-band luminosity (group $L_{r}$ ) to perform the abundance matching and $L_{r}$ correlates closely with cold baryonic mass (K13). Dynamical estimates are shown only for groups with $N>4$ members and show larger scatter with baryonic content at lower halo masses due to having fewer members for the dynamical mass calculation.

content of groups and that every halo is populated by a galaxy. The first assumption implies a monotonic relationship between the HAM group halo mass and group $M_{\text {star }}$ or $M_{\text {bary }}^{\text {cold }}$ as seen in Figure 1(a). This assumption will fail in the presence of significant variations in the hot baryon fraction.

\subsubsection{Group Dynamical Masses}

For groups with multiple members, we compute dynamical masses using the relative velocities and projected distances of the galaxies from the group center. We use the virial theorem to calculate the dynamical mass,

$$
\hat{M}_{\text {halo }}^{\text {dyn }}=A \frac{\sigma_{\text {grp }}^{2} R_{\text {proj }}}{G},
$$

where $\sigma_{\mathrm{grp}}$ is the velocity dispersion of the group, $R_{\mathrm{proj}}$ is the projected radius of the group, and $A$ is a multiplicative scale factor. The scale factor accounts for the projected radius not being the virial radius.

To measure $\sigma_{\mathrm{grp}}$, we use the Gapper method (Beers et al. 1990), which is more robust than a simple rms for low$N$ groups. The Gapper method weights the radial velocities of the galaxies in each group using the formula

$$
\sigma_{\mathrm{Gapper}}=\frac{\sqrt{\pi}}{n(n-1)} \sum_{i=1}^{N-1} \Delta v_{i} w_{i}
$$

where $N$ is the number of galaxies in the group, $\Delta v_{i}$ is $v_{i+1}-v_{i}$ (the velocities have been ordered from smallest to largest), and $w_{i}=i(N-i)$.

The group's projected radius is measured relative to the group center computed by taking the mean of the member galaxies' R.A. and decl. coordinates. Using the technique from Robotham et al. (2011), we order the galaxies' projected radii from the center from smallest to largest, and assign to each ordered radius the percentage of galaxies within that radius $(0 \%-100 \%)$. We then find the radius corresponding to the 75 th percentile to be the projected radius (thus, the two galaxies with percentiles bracketing 75 determine the group radius). We use a larger percentile than the preferred 50th percentile used in Robotham et al. (2011), which best recovered group radii for $N>20$ groups but also developed artifacts for low- $N$ systems due to group-finding errors and the group radius definition. These artifacts are reduced when using a larger percentile definition, although choosing too large a percentile will result in projected radius measurements that are susceptible to outliers.

The underlying assumption of the dynamical approach to mass estimation is that the group halo is virialized, which may or may not be a safe assumption. To assess the validity of this assumption, we use the Anderson-Darling test (A-D test) following the methods of Hou et al. (2009) to look for whether the distribution of radial velocities is consistent with a Gaussian distribution. The $\mathrm{A}-\mathrm{D}$ test is considered robust for $N \geqslant 5$ systems (D'Agostino \& Stephens 1986), and we find that $\sim 90 \%$ of our groups with $N \geqslant 5$ can be classified as virialized.

In Figure 1 , we show group $M_{\text {bary }}^{\text {cold }}$ versus $M_{\text {halo }}^{\text {HAM }}$ (using group $L_{r}$ ) and versus $\hat{M}_{\text {halo }}^{\text {dyn }}$ for RESOLVE-B and ECO. The built-in relationship between group $M_{\text {bary }}^{\text {cold }}$ and $M_{\text {halo }}^{\text {HAM }}$ is apparent, while the relationship between group $M_{\text {bary }}^{\text {cold }}$ and $\hat{M}_{\text {halo }}^{\text {dyn }}$ shows more scatter, especially toward lower-mass groups that have fewer galaxies with which to compute the dynamics. We scaled $\hat{M}_{\text {halo }}^{\text {dyn }}$ by $A=9.9$, which minimizes the offset between HAM and dynamical group mass estimates for groups with $N>7$. The HAM masses already match the simulation HMF by construction.

\subsubsection{Group Dynamical Masses Through Stacking}

For low- $N$ groups, dynamical masses are less reliable, and for singleton and pair groups, they are impossible to calculate. Therefore, for groups with $N>2$ we stack groups of similar properties and compute the velocity dispersion and projected radius from a larger number of galaxies.

To compute the stacked dynamical masses, we first need to determine what group properties to stack on. For the first parameter, we used the group-integrated luminosity, which to 

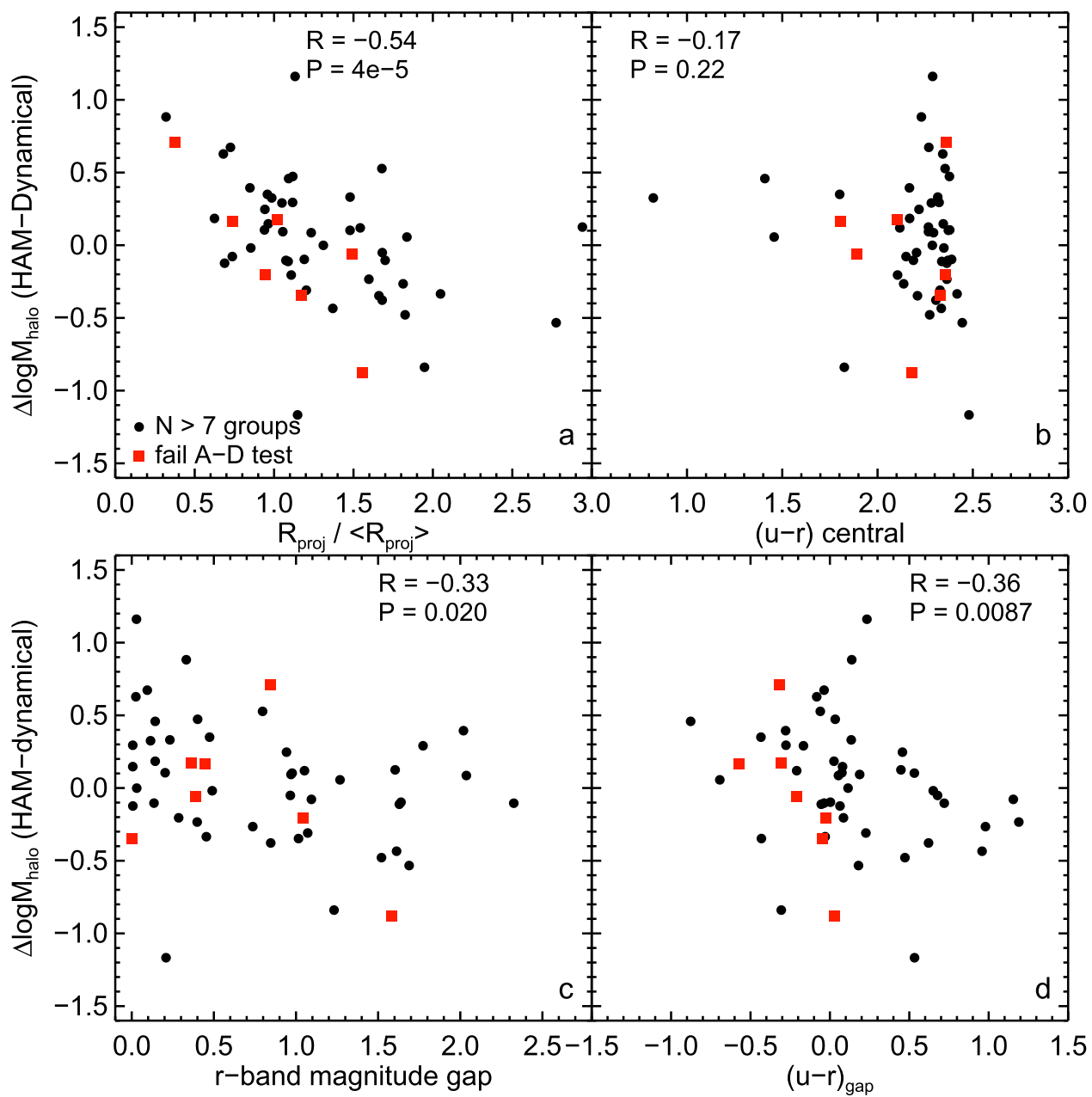

Figure 2. Residual correlations between $\Delta \log M_{\text {halo }}$ and (a) $\left.R_{\text {proj }} /<R_{\text {proj }}\right\rangle$, the projected radius normalized to the median projected radius at a given group $L_{r}$, (b) $u-r$ color of the central, (c) $r$-band magnitude gap, and (d) $u-r$ color gap. Only groups with $N>7$ members are shown, and $\Delta \log M_{\text {halo }}$ is computed as the difference between the $\log$ of $M_{\text {halo }}^{\mathrm{HAM}}$ and the $\log$ of $\hat{M}_{\text {halo }}^{\text {dyn }}($ scaled by $A=9.9$ ). Groups that fail the A-D test (i.e., those that are not virialized) are shown in red. The Spearman rank correlation coefficient and probability of no correlation are reported as $R$ and $P$, respectively. $R_{\text {proj }} /<R_{\text {proj }}>$ yields the most significant correlation with $\Delta \log M_{\text {halo }}$ with the highest coefficient of correlation and the least scatter. The color of the central satellite does not show a significant correlation with $\Delta \log M_{\text {halo }}$. The $r$-band magnitude gap and $u-r$ color gap do show significant correlations with $\Delta \log M_{\text {halo, }}$ although with smaller coefficients of correlation and greater scatter than for $R_{\text {proj }} /$ $<R_{\text {proj }}>$.

first order should track the mass of the group. For the second parameter, we tested different quantities that may relate to the dynamical state of the group by examining whether they correlate with residuals from the relation between $M_{\text {halo }}^{\mathrm{HAM}}$ and $\hat{M}_{\text {halo }}^{\text {dyn }}$ in $N>7$ member groups (Figure 2). Typically, a cutoff of $N=10$ is used for reliable dynamical mass measurements; however, we chose $N>7$ because the distribution between the two variables is roughly Gaussian in this regime and we can increase the sample of groups from 34 to 51 . The parameters are the projected radius normalized to the median projected radius for a given group $L_{r}\left(R_{\text {proj }} /\left\langle R_{\text {proj }}\right\rangle\right), u-r$ color of the central galaxy, $r$-band magnitude gap, and $u-r$ color gap. The last two are computed as the difference in the quantity between the central (the brightest galaxy in $M_{r \text {,tot }}$ ) and the brightest satellite galaxy (the second brightest galaxy in $M_{r \text {,tot }}$ ).

The normalized projected radius may reveal offsets in dynamical mass to the extent that the degree of compactness relates to dynamical status. Since $R_{\text {proj }}$ goes into the dynamical mass measurements, there is a covariance between this quantity and the halo mass residuals. The magnitude gap between the central and brightest satellite galaxies has been used as a tool to detect groups and clusters that assembled early and hence are more dynamically relaxed (e.g., Ponman et al. 1994; Jones et al. 2003). The recent merger history of halos, however, may enhance or diminish magnitude gaps within groups, making them less reliable as an indicator of early assembly (von BendaBeckmann et al. 2008; Dariush et al. 2010). More recent work has examined the use of galaxy color to perform age distribution matching (along with HAM; e.g., Hearin \& Watson 2013), suggesting that the color of the central galaxy or of the entire group may be useful for quantifying the assembly history of the group. Based on these studies, we explore the central galaxy color as well as a quantity that we call the color gap, which is the difference in color between the central and brightest satellite galaxies.

We find that $R_{\text {proj }} /\left\langle R_{\text {proj }}\right\rangle$ yields the most significant correlation with $\Delta \log M_{\text {halo }}$ using the Spearman rank correlation test, with a correlation coefficient of -0.54 and relatively little scatter. The $r$-band magnitude gap and $u-r$ color gap also yield significant correlations with the halo mass residuals, although both have small correlation coefficients compared to $R_{\text {proj }} /\left\langle R_{\text {proj }}\right\rangle$. It is interesting that the color gap yields such a significant correlation (albeit with large scatter), as it suggests 

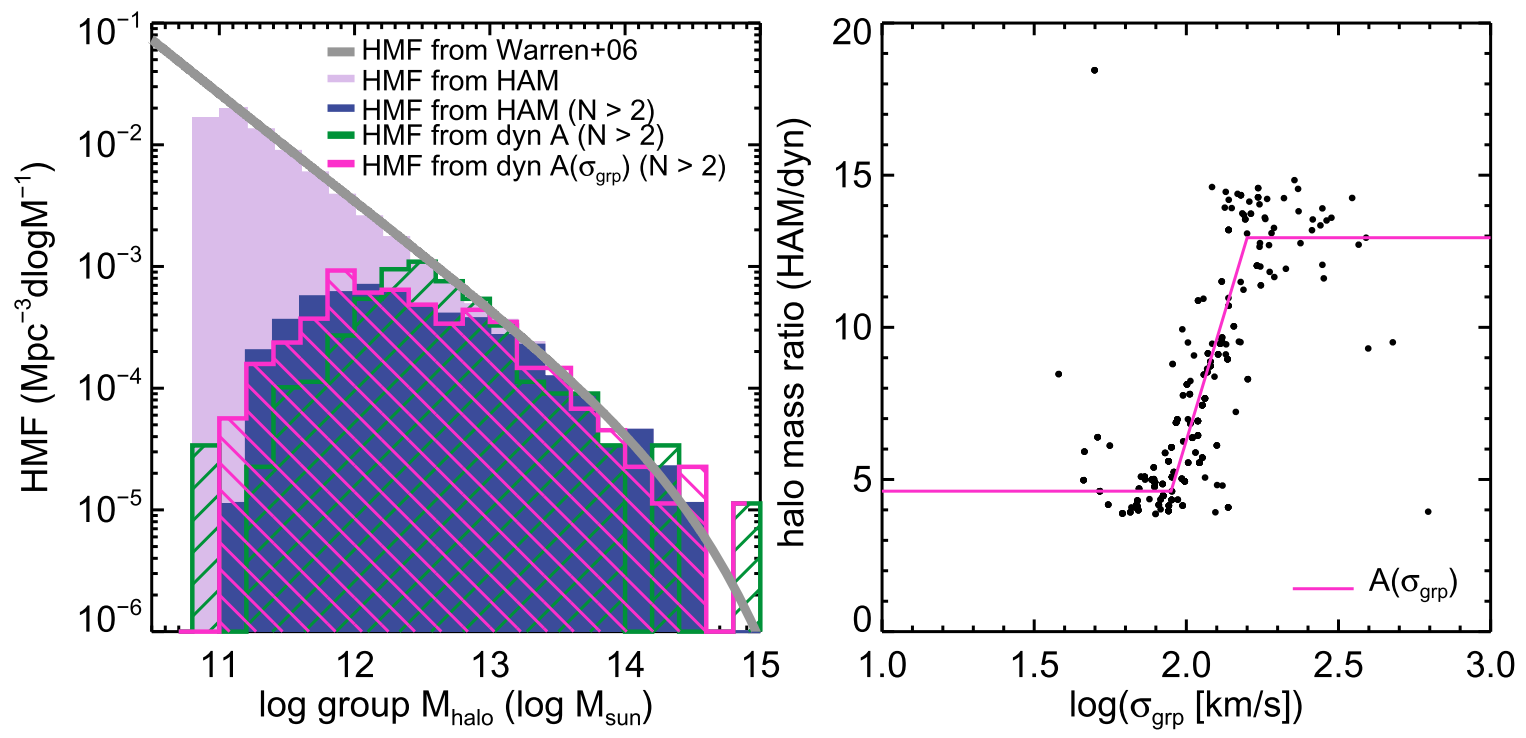

Figure 3. Method to determine the scale factor $A\left(\sigma_{\text {grp }}\right)$ for the dynamical mass estimates (using both direct and stacking estimates). (a) The ECO HMF for $M_{\text {halo }}^{\text {HAM }}($ light purple filled histogram) matches the Warren et al. (2006) HMF (gray thick line) by construction. The ECO HMF for $M_{\text {halo }}^{\text {HAM }}$ for $N>2$ groups only (dark purple filled histogram) is shown for comparison to the HMF of dynamical masses. The HMF of $\hat{M}_{\text {halo }}^{\text {dyn }}$ (scaled by a constant factor of $A$; green cross-hatched histogram) overproduces intermediate-mass groups of mass $\sim 10^{12.5} M_{\odot}$. The HMF of $M_{\text {halo }}^{\mathrm{H} \text {-dyn }}$ scaled by $A\left(\sigma_{\mathrm{grp}}\right)$ (pink cross-hatched histogram) is calibrated to reproduce the cumulative HMF for $N>2$ groups. (b) To determine the scale factor A $\left(\sigma_{\mathrm{grp}}\right)$, we find the HAM and dynamical halo masses at each group's cumulative number density and plot their ratio as a function of $\log \left(\sigma_{\text {grp }}\right)$. Since we do not want to account for groups of $N=1$ and 2, we use the cumulative HMF from the HAM estimates and join it to the cumulative HMF from theory at high masses (where ECO has less data). To fit the data, we take the median of the halo mass ratio at high and low sigma (where the relationship is relatively flat) and fit a line between $\log \left(\sigma_{\mathrm{grp}}\right)=1.9-2.2$ (pink). This calibration is applied to the dynamical mass estimates.

that deviations between the dynamical and HAM mass may be related to deviations from galaxy conformity, the empirical result showing that satellite galaxies tend to have colors and star formation histories similar to their group central galaxies (Weinmann et al. 2006).

We use $R_{\text {proj }} /<R_{\text {proj }}>$ along with group $L_{r}$ to stack $N>2$ groups in bins of 0.2 and $0.25 \mathrm{mag}$, respectively. We then compute the stacked group dynamical mass for each bin, which is applied to all groups in that bin.

\subsubsection{Final Dynamical Group Mass Estimates}

To determine the final dynamical mass estimates, we rely on a combination of measured and stacked estimates at high $N$ and stacked and HAM estimates at low $N$. We also calibrate the hybrid dynamical masses to match the cumulative HMF. We label this hybrid dynamical mass $M_{\text {halo }}^{\mathrm{H}-\text { dyn }}$ to distinguish it from the directly measured dynamical masses that have been scaled by a single constant $\hat{M}_{\text {halo }}^{\text {dyn }}$.

For groups with $N \geqslant 15$, we use the measured dynamical mass. For groups with $3 \leqslant N \leqslant 7$, we use the stacked dynamical mass estimates. For groups with $N$ between 7 and 15 , we transition smoothly between these two regimes by using a linear combination of the stacked dynamical mass estimate and the directly measured dynamical estimate as given by Equation (3):

$$
M_{\text {halo }}^{\mathrm{H}-\mathrm{dyn}}=a \times M_{\text {halo }}^{\mathrm{dyn}}+(1-a) \times M_{\text {halo }}^{\mathrm{dyn}, \text { stack }},
$$

where $a$ (Equation (4)) is a linear function of $N$ such that $a=0$ at $N=7$ and $a=1$ at $N=15$,

$$
a=0.125 N-0.875 \text {. }
$$

We chose $N=15$ as our upper cutoff, as dynamical masses for groups with $N \geqslant 15$ are very reliable. We chose $N=7$ as our lower cutoff as the directly measured dynamical masses down to $N=7$ still show roughly symmetric scatter with HAM halo masses as described in Section 2.3.4. For $N<7$, the scatter becomes asymmetric, and we must rely on the stacked dynamical masses completely.

Before comparing with the HAM masses and addressing the low- $N$ systems further, we must calibrate our dynamical halo masses. In Figure 3(a), we show the theoretical HMF of Warren et al. (2006) as a gray line and the HMF of the ECO $M_{\text {halo }}^{\mathrm{HAM}}$ as a light purple histogram (matched to the theoretical HMF by definition). We also show the $M_{\text {halo }}^{\text {HAM }} \mathrm{HMF}$ for $N>2$ groups as a dark purple histogram. The $\hat{M}_{\text {halo }}^{\text {dyn }} \mathrm{HMF}$ for $N>2$ groups using a constant scale factor of $A$ is shown as the green crosshatched histogram, which overproduces intermediate-mass groups near $\sim 10^{12.5} M_{\odot}$. Since we do not necessarily think that the characteristic group radius should stay the same as a function of group mass, we determine a scale factor $A\left(\sigma_{\mathrm{grp}}\right)$ that preserves the theoretical cumulative HMF. To do this, we perform HAM between the cumulative mass function of the raw dynamical masses and the cumulative HMF (combining the ECO $N>2 M_{\text {halo }}^{\mathrm{HAM}} \mathrm{HMF}$ at low masses with the theoretical $\mathrm{HMF}$ at high masses). We then plot the ratio of the abundancematched halo masses to the raw dynamical masses as a function of group velocity dispersion. The fit to the data is shown in pink in Figure 3(b), and we use this $A\left(\sigma_{\mathrm{grp}}\right)$ scale factor to create the $M_{\text {halo }}^{\mathrm{H}-\text { dyn }} \mathrm{HMF}$ for $N>2$ groups (pink cross-hatched histogram), which better reproduces the theoretical $(N>2)$ $\mathrm{HMF}$ than using the constant value of $A$. At large halo mass, we note that the ECO dynamical mass HMF overpredicts groups relative to the theoretical HMF. These are the few largest clusters in ECO (including the Coma cluster) and thus their number densities are highly subject to cosmic variance.

To incorporate HAM masses at low $N$, we construct a linear combination of the HAM and stacked dynamical masses for groups with $3 \leqslant N \leqslant 5$, increasing the contribution from the 

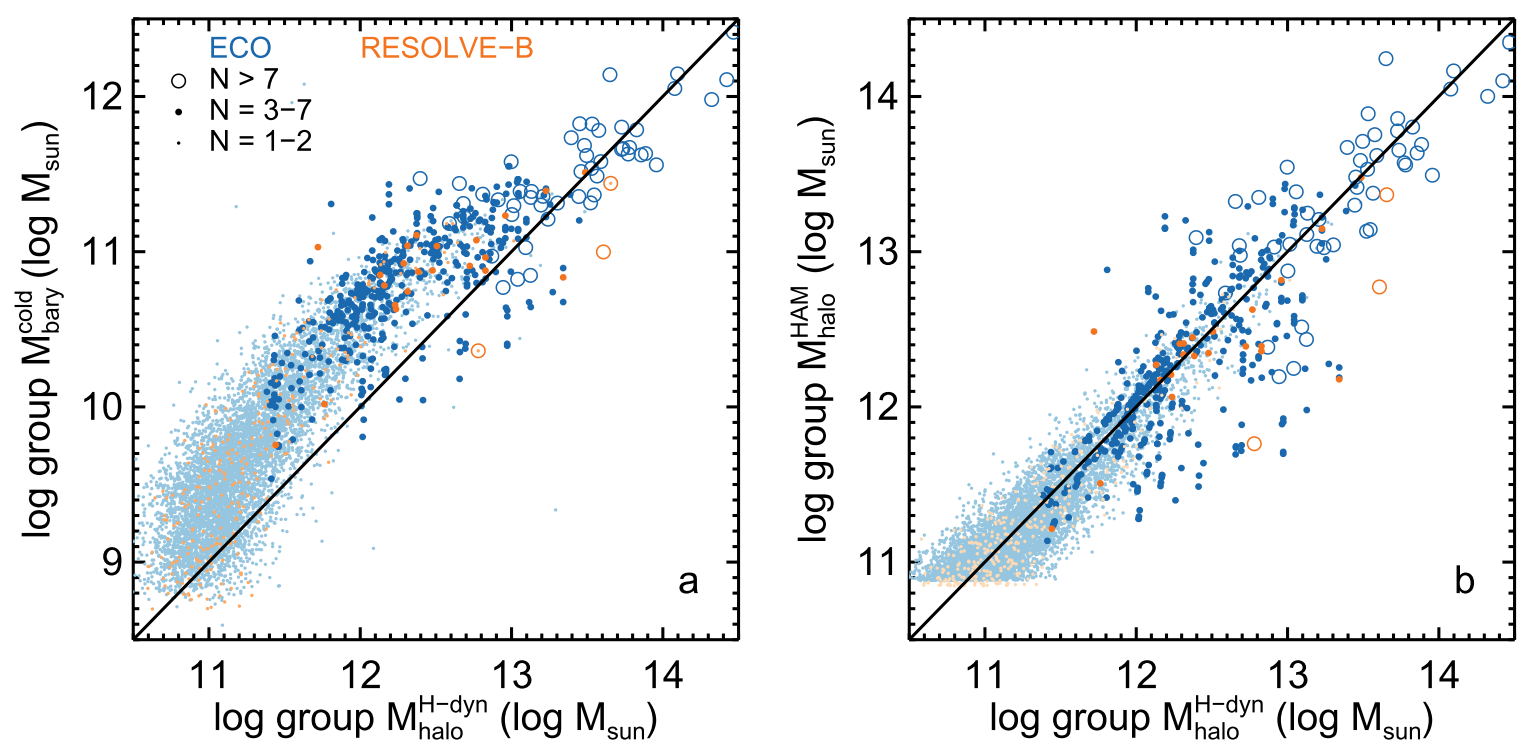

Figure 4. (a) Group $M_{\text {bary }}^{\text {cold }}$ (including satellite completeness corrections) and (b) $M_{\text {halo }}^{\mathrm{HAM}}$ vs. $M_{\text {halo }}^{\mathrm{H} \text {-dyn }}$ using direct and stacking measurements at large $N$ and stacking measurements and HAM at low- $N$. ECO is shown in blue and RESOLVE is shown in orange. The black line shows a one-to-one relationship shifted down by 2 dex in panel (a) and a one-to-one relationship in panel (b). Groups with $N>7$ are shown as open circles, while groups with $3 \leqslant N \leqslant 7$ are shown as medium-sized dots, and groups with $N=1$ and 2 are shown as small dots. Groups are assigned mass according to group $N$ as described in Section 2.3.5.

stacked dynamical masses as a function of $N$. For groups with $N=1$ and 2, we must rely solely on the HAM mass estimate. We note that the scatter in group cold baryonic mass to HAM mass is $\sim 0.14$ dex over the group baryonic mass range of $10^{10.5}-10^{11.5} M_{\odot}$ (after removing the relationship with a second-order polynomial), while the scatter in group cold baryonic mass to dynamical mass over the same group baryonic mass range is $\sim 0.32 \mathrm{dex}$. The larger scatter relative to the dynamical mass is partially due to measurement uncertainties, although the smaller scatter for HAM masses is built in due to the tightness in the correlation between group $L_{r}$ and cold baryonic mass (K13). To assess the contribution from measurement uncertainty to the dynamical mass scatter, we determine the error on $\sigma_{\text {grp }}$ for groups with $N>15$. We also determine the uncertainty due to projection effects on $\sigma_{\text {grp }}$ and $R_{\text {proj }}$ by examining the scatter in $\sigma_{\mathrm{grp}}$ and $R_{\text {proj }}$ at fixed $\sigma_{\mathrm{grp}}$ and $R_{\mathrm{vir}}$ for known groups in a mock catalog (the same mock catalog used in the Appendix). By propagating these uncertainties through the measurement of dynamical masses, we find that the typical measurement error on the dynamical mass is $\sim 0.22$ dex. In this analysis of uncertainty, we excluded group-finding errors, which also affect the HAM masses. Taking the quadrature difference between the measured scatter (0.32 dex) and the measurement uncertainty ( $0.22 \mathrm{dex})$, we find that the intrinsic scatter in dynamical mass is likely closer to 0.23 dex. Thus, to create a smooth transition from $N=1$ and 2 groups to $N \geqslant 3$, we match the scatter in HAM masses of $N=1$ and 2 groups to 0.23 by adding 0.18 dex scatter (the quadrature sum of 0.18 and 0.14 is 0.23 ).

In Figure 4, we show group $M_{\text {bary }}^{\text {cold }}$ versus group $M_{\text {halo }}^{\mathrm{H}-\text { dyn }}$ and group $M_{\text {halo }}^{\mathrm{HAM}}$ versus group $M_{\text {halo }}^{\mathrm{H} \text {-dyn }}$ for all groups. $M_{\text {halo }}^{\mathrm{H} \text {-dyn }}$ combines the direct and stacked dynamical mass estimates and HAM mass estimates with scatter into one group mass variable. We note that the curvature between halo mass and groupintegrated cold baryonic mass seen in Figure 1 using the HAM group halo masses is also apparent in Figure 4(a) when using the dynamical group halo masses, albeit with larger scatter.
Examining the scatter in Figure 4(b) in greater detail, we note that at fixed $M_{\text {halo }}^{\mathrm{H}-\text { dyn }}$ the scatter in $M_{\text {halo }}^{\mathrm{HAM}}$ abruptly decreases below $10^{12} M_{\odot}$. At fixed $M_{\text {halo }}^{\mathrm{HAM}}$, we find that the scatter in $M_{\text {halo }}^{\mathrm{H} \text {-dyn }}$ below $10^{12} M_{\odot}$ is asymmetric, with greater scatter toward higher dynamical mass than lower dynamical mass. These scatter trends highlight the limitations of our data set as we go to lower group masses where groups have fewer galaxies with which to accurately measure dynamical masses. They also suggest, however, that there is greater scatter between cold baryonic content within low-mass groups than is evident from HAM group mass estimates.

\subsection{Group Completeness Corrections for ECO}

In Section 2.1, we discussed the galaxy completeness corrections computed for ECO to account for galaxies that are bright enough to be included in our survey, but were missed due to either fiber collisions or photometry issues. These completeness corrections are produced to account for galaxies above our survey absolute magnitude limit. We extend these galaxy completeness corrections to group completeness corrections with the following simple algorithm.

The largest completeness corrections are for low-luminosity galaxies, which are generally either satellites of larger groups or low-mass galaxies in $N=1$ groups. Therefore, we consider two types of completeness corrections for groups: corrections for satellites, which affect the mass of the group, and corrections for centrals, which affect the number density of groups.

For satellite completeness corrections, we use each satellite's galaxy completeness correction to compute the weighted sum of either stellar or cold baryonic mass in producing group $M_{\text {star }}$ and $M_{\text {bary }}^{\text {cold }}$. The central galaxy is automatically given a completeness correction of 1.0 (no correction). Typically, the group-integrated stellar and cold baryonic masses are increased by $\leqslant 5 \%-10 \%$. To include these satellite completeness corrections in the group stellar and baryonic mass distributions computed in Section 2.3.1, we scale the mass distribution of 
each satellite by its completeness correction before performing the pseudo-convolution.

For central completeness corrections, we use the galaxy completeness correction of each group central to weight the group mass functions and density fields presented in Section 3. In this case, we expect to miss $N=1$ groups, but not large groups. Indeed, we find that the central completeness corrections increase the number of $N=1$ groups by $\sim 18 \%$, while they increase the number of $N>7$ groups by $\sim 3 \%$.

These group completeness corrections have not been explicitly accounted for in the group-finding, HAM, or dynamical mass measurements. Truly accounting for the missing galaxies, however, would affect all three. We note that increasing the number densities of low-mass groups will systematically shift the HAM masses to lower masses. We also note that the satellite completeness corrections increase the group-integrated masses by $\leqslant 5 \%-10 \%$, which will also affect HAM mass estimates. For dynamical mass estimates, we can only rely on the galaxy data available to estimate velocity dispersions.

\subsection{The Semi-analytic Model Mock Catalog}

To compare our results with models of galaxy evolution, we create a mock catalog based on the SAM described in Gonzalez-Perez et al. (2014), which builds on the Lagos et al. (2012) model. This SAM is a variant of the GALFORM model (Cole et al. 2000) and is particularly relevant to this work because it calculates separately the cold atomic and molecular gas components, which enables both implementation of more realistic star formation prescriptions using only the molecular gas component (Lagos et al. 2011a, 2011b) and direct comparison with our cold atomic gas data.

The GALFORM model starts with the dark-matter-only Millennium simulation halo merger trees (Springel et al. 2005). This particular SAM uses the Millennium run with the WMAP7 cosmology $\left(\Omega_{m}=0.27, \Omega_{\Lambda}=0.728, H_{0}=70.4\right.$ $\mathrm{km} \mathrm{s}^{-1}$; Komatsu et al. 2011). The formation and evolution of galaxies are built on top of the dark-matter-only foundation by adding gas to halos and following prescriptions for gas heating (in the form of shocks and feedback from stars and active galactic nuclei, AGNs), gas cooling, star formation, metal enrichment, and black hole formation. We refer the reader to Gonzalez-Perez et al. (2014) for an in-depth description of these processes. We note, however, that the SAM used in this work differs from that of Gonzalez-Perez et al. (2014) in its treatment of a galaxy's hot gas once it becomes a satellite within a larger halo. In Gonzalez-Perez et al. (2014), the satellite's hot gas is immediately stripped upon entering the halo. In the SAM used in this work, a ram pressure stripping algorithm described in Lagos et al. (2014) gradually removes the satellite's hot gas. The gradual stripping of gas results in higher cold gas fractions in early-type galaxies due to the continuing accretion of gas from the satellite's cooling hot halo, in better agreement with observations (Lagos et al. 2014). Although this change in hot gas stripping of satellites clearly affects the galaxy cold-baryon content, it is less certain how it should affect the group $M_{\text {bary }}^{\text {cold }}$, as the stripped satellite hot gas could still cool onto the central galaxy. The cooling of gas depends on the cooling and dynamical timescales of the (sub)halo, and thus gas that may have cooled onto the satellite may not cool onto the central galaxy. It should be noted that the ram pressure stripping here removes the hot gas and not the cold gas as originally described in Gunn \& Gott (1972). Although ram pressure stripping is an effective means of removing galaxy cold gas, it primarily affects satellites of the largest clusters, of which there are relatively few in RESOLVE and ECO.

For a direct comparison to the RESOLVE-B and ECO data sets, we produced a mock catalog from the $z=0$ output of the SAM by converting the positions and velocities of its galaxies to R.A., decl., and redshift. To compute R.A. and decl., we convert from Cartesian to spherical coordinates with the origin placed at the center of the box. To compute the redshift, we measure the distance to each galaxy from the origin and obtain the cosmological redshift using a Hubble constant of $70 \mathrm{~km} \mathrm{~s}^{-1} \mathrm{Mpc}^{-1}$. The redshift velocity is added to the velocity of the galaxy within the simulation, which corresponds to its peculiar velocity.

The SAM mock catalog extends to $c z=15,000 \mathrm{~km} \mathrm{~s}^{-1}$, although we cut down the volume to a spherical shell extending between $c z=2530-7470 \mathrm{~km} \mathrm{~s}^{-1}$ and select galaxies brighter than $M_{r}=-17.33$ (similar to ECO). To ensure that the SAM absolute magnitudes are roughly consistent with the reprocessed magnitudes for ECO, we examine the $r$-band luminosity function for ECO (completeness corrected) and for the entire $z=0 \mathrm{SAM}$ box (for greater statistics than the smaller mock catalog). The SAM luminosity function has a shape similar to that of ECO, albeit offset toward fainter magnitudes. Based on this comparison, we shifted the SAM magnitudes brighter by $\sim 0.2 \mathrm{mag}$ to be consistent with the ECO luminosity function near $M_{r \text {,tot }}=-23.0$, where the ECO luminosity function reaches 10 galaxies per $\mathrm{Mpc}^{3}$. The final mock volume is $\sim 1,649,480 \mathrm{Mpc}^{3}$ and has an overall number density of galaxies brighter than -17.33 of $\sim 0.0233 \mathrm{Mpc}^{-3}$ (similar to the completeness-corrected ECO number density of $\sim 0.0247 \mathrm{Mpc}^{-3}$ ).

We perform FOF group finding and HAM for the SAM using the same codes as described in Section 2.3 so that we can examine both the "true" groups and the "FOF" groups that would be identified by an observer with group-finding errors. We perform the same algorithm to break up false pairs as was used for the ECO and RESOLVE-B data sets. To reduce errors in group finding due to galaxies with large peculiar velocities, we further cut down the mock to galaxies with group redshifts within a spherical shell $3000-7000 \mathrm{~km} \mathrm{~s}^{-1}$ (similar to ECO). Within this smaller volume, there are 65,784 true groups $(57,109$ are $N=1)$ and there are 69,161 FOF groups $(58,587$ are $N=1)$.

For both the true and FOF groups, we measured the group $M_{\text {star }}$ and $M_{\text {bary }}^{\text {cold }}$. We note that the IMF used in the model is that of Kennicutt (1983b), which is different from the Chabrier (2003) IMF used to compute stellar masses for RESOLVE-B and ECO. The two IMFs yield similar mass-to-light ratios, so we do not expect this difference to cause significant systematics in comparing the model and the data. For cold baryonic mass, we sum the stellar and cold atomic gas mass in each galaxy, for which cold atomic gas mass is defined as $1.4 M_{\mathrm{H}}$ to account for helium. Although the SAM records the exact amount of $\mathrm{HI}$ mass in each galaxy, our observations frequently allow us to constrain the gas mass as an upper limit of $\sim 5 \%-10 \%$ of the galaxy's stellar mass. To reflect this observational effect on the SAM, we replace the gas mass with $0.05 M_{\text {star }}$ if its value is less than $5 \%$ of the stellar mass.

To ensure that the SAM true and FOF group halo masses are consistent with the ECO data, we compare the HMFs. We find 

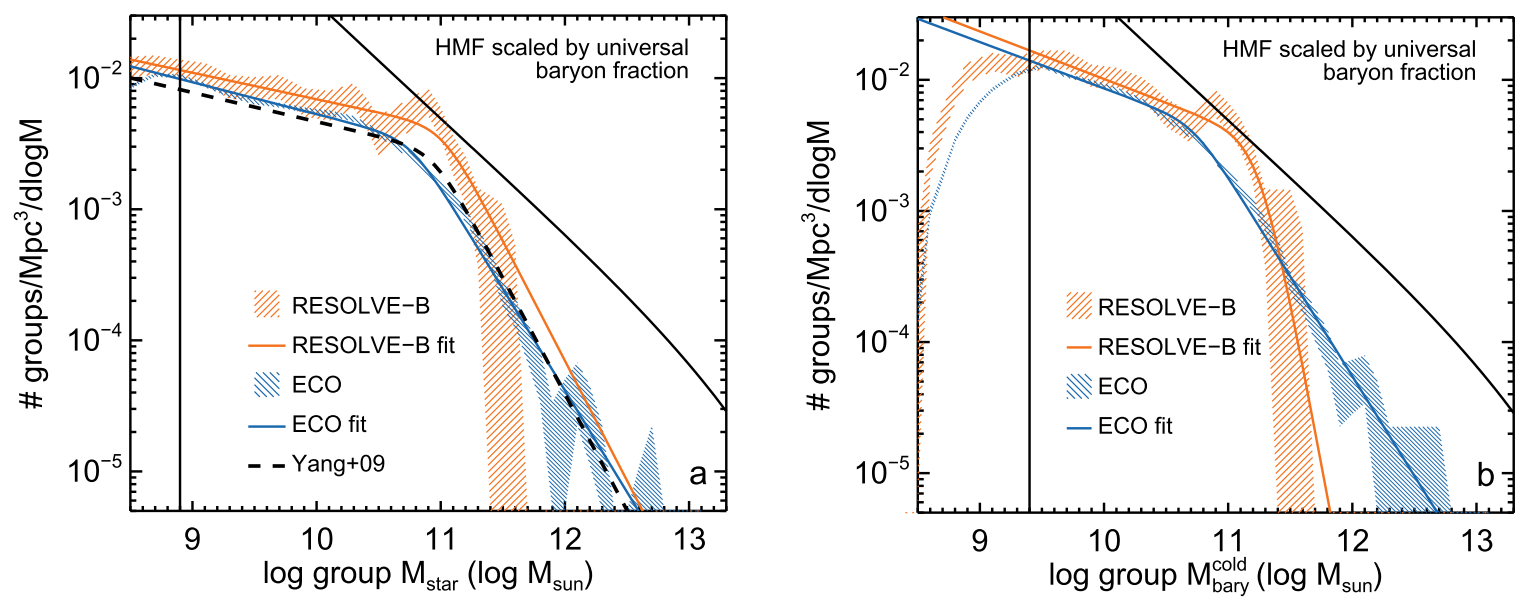

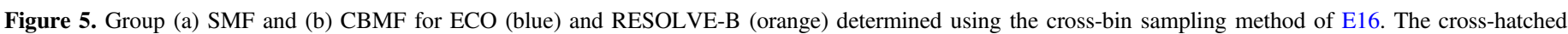

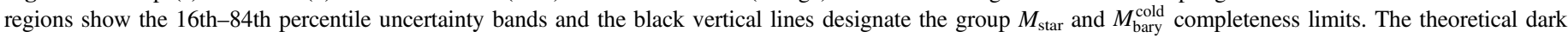

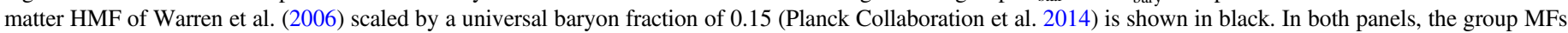

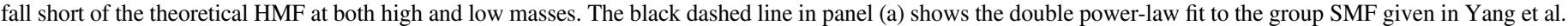

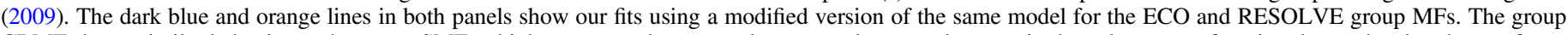

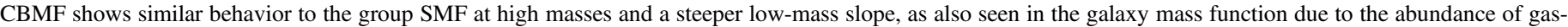
dominated galaxies at low halo masses (E16).

no significant offset and thus we apply no correction to the group halo masses.

\section{Group Mass Functions and Baryon Fractions}

We now examine the group-integrated stellar and cold baryonic mass functions (or group SMF and CBMF) and group-integrated stellar and cold-baryon fractions for the RESOLVE-B and ECO data sets as well as for the SAM mock catalog.

\subsection{Group Mass Functions}

To measure the group SMF and CBMF for RESOLVE-B and ECO, we adapt the cross-bin sampling technique described in E16. For the galaxy MFs in E16, this method first combines all individual mass likelihood distributions into one combined survey mass likelihood distribution by summing the likelihoods in each bin. Then, the overall stellar or baryonic mass functions are constructed by sampling from the combined survey mass likelihood distribution 1000 times in a Monte Carlo fashion. From these 1000 samples, we determine the median and the uncertainty bands (16th-84th percentiles of the mass functions). In this work, rather than constructing the mass likelihood distribution of all of the galaxies in our data set, we construct the mass likelihood distribution of all of the groups in our data set using the individual group mass likelihood distributions computed in Section 2.3.1. As in E16, the likelihoods for ECO are weighted by the central completeness correction factor (described in Section 2.4).

The group SMFs for RESOLVE-B and ECO are shown in Figure 5(a). The fact that RESOLVE-B is elevated over ECO is due to cosmic variance. In E16, we found that RESOLVE-B is overdense compared to ECO at intermediate and low galaxy masses, although it is underdense compared to ECO at large masses due to a lack of cluster-sized halos. Figure 5(a) also compares the group SMF with the HMF from Warren et al. (2006), which does not include the contribution from subhalos and is scaled by a universal baryon fraction of 0.15 (Planck Collaboration et al. 2014) for direct comparison with the data.
We also use this HMF to assign HAM halo masses to the data and SAM mock catalog.

At high masses, the group SMF drops off more steeply than the universal baryon fraction scaled HMF. Around $M_{\text {star }}=10^{10.9} M_{\odot}$ the group SMF reaches a maximum compared to the HMF, which we determine by finding the group $M_{\text {star }}$ at the maximum of the ratio between the group $\mathrm{SMF}$ and the universal baryon fraction scaled HMF. Below $10^{10.9} M_{\odot}$, the group SMF exhibits a shallow rise, even shallower than the galaxy SMF (not shown; see E16). This slow rise reflects the fact that low-mass satellites from the galaxy SMF are removed from the low-mass end and placed into high-mass groups in the group SMF. This result clearly illustrates the large gap between the group SMF and dark matter HMF at low group-integrated stellar masses.

To compare with previous work, we overplot the double power-law fit to the group SMF from Yang et al. (2009) in Figure 5(a) as a black dashed line. They used the Yang et al. (2007) group catalog constructed from the NYU-VAGC SDSS galaxy catalog (Blanton et al. 2005b). Their group-finding algorithm used FOF to define potential groups and then an iterative process to assign galaxies to each potential group based on its mass and size. To fit their group SMFs, they used the equation

$$
\phi(m)=\phi_{*} \frac{\left(\frac{m}{M_{*}}\right)^{\alpha}}{\left(x_{0}+\left(\frac{m}{M_{*}}\right)^{4}\right)^{\beta}}
$$

and found the parameters $\phi_{*}=0.00731, \log M_{*}=10.67, x_{0}=$ $0.7243, \alpha=-0.2229$, and $\beta=0.3874$.

We performed fits using a modified version of this double power-law model, fixing $x_{0}=1$ since this parameter is not well-constrained by our data. We find that fixing $x_{0}$ affects the values of $\phi_{*}$ and $\log M_{*}$ but not $\alpha$ and $\beta$. Our own fits using this modified double power-law model are shown by the solid orange and blue lines for RESOLVE-B and ECO, respectively, and the model parameters are given in Table 2. We find a similar low-mass slope for both RESOLVE-B and ECO of $\alpha \sim-0.2$ to that found in Yang et al. (2009). At higher group 
Table 2

Fitting Parameters for the Group-integrated Mass Function

\begin{tabular}{|c|c|c|c|c|}
\hline Data & $d \log M^{\phi_{*}} \mathrm{Mpc}^{-3}$ & $\begin{array}{c}\log M_{*} \\
\log \left(M_{\odot}\right)\end{array}$ & $\alpha$ & $\beta$ \\
\hline RESOLVE-B group SMF & $0.0043 \pm 0.0011$ & $11.02 \pm 0.28$ & $-0.20 \pm 0.05$ & $0.41 \pm 0.31$ \\
\hline ECO group SMF & $0.0035 \pm 0.0002$ & $10.76 \pm 0.04$ & $-0.24 \pm 0.01$ & $0.33 \pm 0.02$ \\
\hline RESOLVE-B group CBMF & $0.0035 \pm 0.0018$ & $11.26 \pm 0.46$ & $-0.36 \pm 0.07$ & $1.17 \pm 3.25$ \\
\hline ECO group CBMF & $0.0048 \pm 0.0003$ & $10.73 \pm 0.04$ & $-0.35 \pm 0.02$ & $0.29 \pm 0.02$ \\
\hline
\end{tabular}

masses, we observe slight differences in the group SMF knee and high-mass falloff between ECO and the fit from Yang et al. (2009), although these are likely within systematic uncertainties between studies such as the stellar mass estimation and cosmic variance. For RESOLVE-B, the double power-law model does not fit the data well at high masses, as can be seen by the fit as well as the large errors on the $\beta$ parameter, which describes the power-law slope of the high-mass end. The poor fit at high masses is likely due to the fact that the RESOLVE-B subvolume is small (i.e., highly subject to cosmic variance) and has no large clusters of mass $>10^{13.5} M_{\odot}(\mathrm{E} 16)$, resulting in a very steep dropoff at high group masses. It was further shown in E16 that it is possible to reconstruct the RESOLVE-B galaxy mass function by scaling a set of basis conditional mass functions (galaxy mass functions broken down into different halo mass regimes) by the number of group halos in each halo mass regime, thus suggesting that a given survey's mass function can be determined by its halo mass distribution.

The group CBMFs for RESOLVE-B and ECO are shown in Figure 5(b). At high mass, they are very similar to the group SMFs, since high-mass groups generally have little cold gas (e.g., Haynes et al. 1984). Indeed, we find that the power-law slopes of the high-mass ends are very similar between the group $\mathrm{SMF}$ and $\mathrm{CBMF}(\beta \sim 0.3$ for $\mathrm{ECO}$; we note that RESOLVE-B is not well-constrained at high masses). At $M_{\text {bary }}^{\text {cold }}=10^{10.9} M_{\odot}$, the group CBMF also reaches its relative maximum to the group HMF (measured again by finding the group $M_{\text {bary }}^{\text {cold }}$ at the maximum of the ratio of the group CBMF to the scaled HMF). Below $10^{10.9} M_{\odot}$, the group $\mathrm{CBMF}$ rises more steeply than the group SMF $\left(\alpha_{\mathrm{SMF}} \sim-0.24\right.$ versus $\left.\alpha_{\mathrm{CBMF}} \sim-0.35\right)$, similar to the galaxy BMF versus SMF shown in E16. However, the group CBMF is still not as steep as the HMF.

At all masses, the group SMF and CBMF fall below the scaled HMF, although perhaps for different reasons. In large groups, we expect that hot gas dominates the baryon content, and we will examine its effect on the group BMF in Section 3.3. At lower masses, the separation from the HMF may also be indicative of unaccounted-for gas (see Section 4.2) or due to other processes related to galaxy and group formation.

In Figure 6, we show the group SMF (magenta) and CBMF (light pink) for the FOF groups in the SAM mock catalog. We note that the results do not change significantly if we use the true groups rather than the FOF groups. Since the galaxy masses are known to precision (albeit with systematic errors due to the assumptions in the model such as the IMF, star formation prescription, and gas definition), we bin the values and assume Poisson error bars for each bin.

At high mass $\left(>10^{11} M_{\odot}\right)$, the group SMF and CBMF for the SAM are similar to the ECO group SMF and CBMF (overplotted in dark and light blue, respectively). Below $\sim 10^{11} M_{\odot}$, however, the SAM group SMF and CBMF deviate significantly from the data. We find that the SAM contains many fewer groups with group $M_{\text {bary }}^{\text {cold }} \lesssim 10^{11} M_{\odot}$. The knee of the SAM group SMF and CBMF is located at higher masses than that observed in ECO. We explore the location of the knee of the group SMF and CBMF and its relation to the feedback implementation in the model in more detail in Section 4.1. The SAM group SMF and CBMF then rise more steeply at the lowest group halo masses.

\subsection{Group Stellar and Cold-Baryon Fractions}

We now examine group-integrated stellar and cold-baryon fractions as a function of halo mass for ECO and RESOLVE-B. These fractions are defined as the group $M_{\text {star }}$ or $M_{\text {bary }}^{\text {cold }}$ divided by $M_{\text {halo }}$. We can interpret these fractions as the stellar or baryonic collapse efficiency of groups, i.e., how many of the group halo baryons have collapsed into the observable stars and cold gas in galaxies. To increase our statistics, we analyze the ECO and RESOLVE-B data sets together, using the group central completeness corrections to weight ECO groups appropriately (see Section 2.4). ${ }^{8}$ Figures 7-9 compare results using HAM and dynamical group mass estimates. The groupintegrated stellar and cold baryonic mass completeness limits are shown as dashed black lines.

Using HAM (Figures 7 and 8 show the results based on matching on the group $L_{r}$ and group $M_{\text {star }}$, respectively), we find that the group stellar and cold-baryon fractions peak around a halo mass of $\sim 10^{11.8} M_{\odot}$, although the cold-baryon fraction peak is much broader than that of the stellar fraction. At higher and lower group halo mass, the stellar and coldbaryon fractions fall off. This behavior has been seen in previous work examining the stellar fraction using either the HAM or HOD method for assigning halo mass (e.g., Leauthaud et al. 2012a). The falloff toward higher group halo masses is interpreted to show that high-mass groups are increasing their hot gas and dark matter content faster than their collapsed cold baryonic mass content. The falloff toward low group halo masses would then correspondingly show that low-mass galaxies (generally single galaxies in their own halo down to our survey limits) are growing rapidly in cold baryonic mass (from cooling halo gas), while their halo mass is not increasing as quickly (Conroy \& Wechsler 2009; Moster et al. 2010). We note that in the largest group mass bin the stellar and coldbaryon fraction rises. This bin, however, consists of one group, whose corresponding dynamical mass does not confirm such a rise as physically meaningful (Figure 9).

An alternative, static interpretation of these plots is that lowmass galaxies have suppressed cold baryonic content due to stellar and supernova feedback. Studies based on simulations suggest, however, that dwarf galaxies with gas masses of

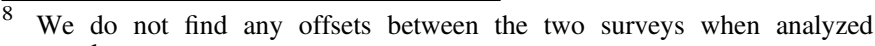
separately.
} 

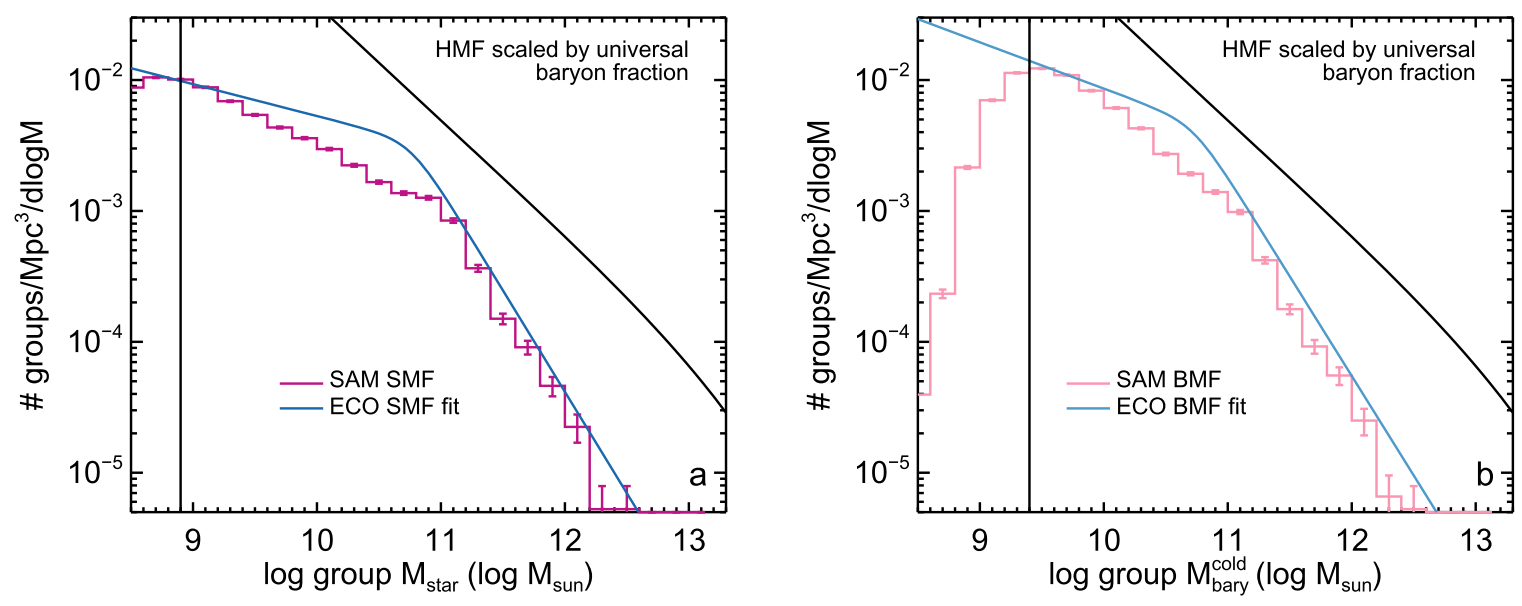

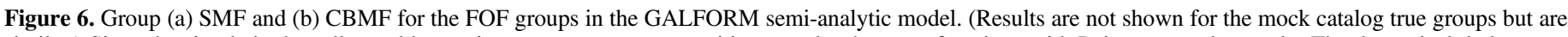

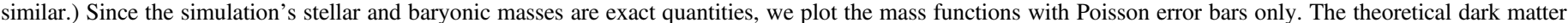

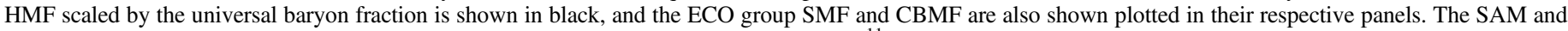

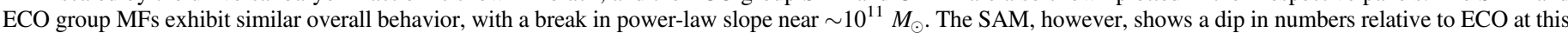

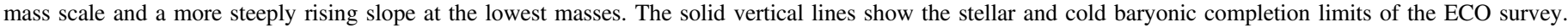

$10^{8}-10^{9} M_{\odot}$ do not lose their gas due to stellar and supernova feedback (Mac Low \& Ferrara 1999; Melioli et al. 2015), and in addition, cooling of fresh and recycled gas in low-mass halos is generally found to be efficient (Lu et al. 2011; AnglésAlcázar et al. 2017). In fact, the isolated dwarfs in this regime are far from static, as they are doubling their stellar masses on $\sim$ Gyr timescales (K13). Reionization at early times may have heated the gas in lower-mass halos, thus delaying their formation relative to higher-mass galaxies, but at present, their growth rates are high. We also note that galaxies below the survey limit could contribute up to $\sim 14 \%$ of the cold baryonic mass in low-mass groups $\left(M_{\text {halo }}<10^{11.4} M_{\odot} ;\right.$ see Section 2.3.1), increasing the cold-baryon fractions by roughly $\sim 0.05$ dex.

In Figure 7, using HAM based on group luminosity, the peak of the stellar mass fraction is sharper than that of the cold baryonic mass fraction, with a steeper falloff toward lower-mass groups. Adding the cold gas results in a flatter peak over the nascent group regime that has little scatter in cold-baryon fraction. In Figure 8, using HAM based on group $M_{\text {star }}$, however, we find that the group cold-baryon fraction becomes much more scattered at low masses, likely due to the fact that HAM based on group $M_{\text {star }}$ does not track cold baryonic mass as well as HAM based on group $L_{\mathrm{r}}$ does in the low group halo mass regime.

In Figure 9, we show the group stellar and cold-baryon fractions as a function of dynamical mass as described in Section 2.3.5. At high group halo masses, the stellar and coldbaryon fractions are similar to results using HAM, decreasing with increasing halo mass. At all masses, however, we find a much greater diversity in stellar and cold-baryon fractions with $M_{\text {halo }}^{\mathrm{H} \text {-dyn }}$ than with $M_{\text {halo }}^{\mathrm{HAM}}$ (e.g., width $\sim 1$ dex versus $\sim 0.5 \mathrm{dex}$ near $M_{\text {halo }}=10^{12} M_{\odot}$ ). Thus, HAM may build in a perceived tight maximum in baryonic collapse efficiency over the nascent group regime, whereas the dynamical masses suggest there should be more scatter, potentially due to variations in the hot gas fractions within groups. We note that Figure 9 includes $N=1$ and 2 groups, which rely purely on HAM (with increased scatter to smoothly transition to $N \geqslant 3$ dynamical masses) for mass estimates.
In Figure 10, we show the stellar and cold-baryon fractions as a function of $M_{\text {halo }}^{\mathrm{HAM}}$ for the SAM FOF mock catalog. Using HAM results in the familiar shape seen in the ECO and RESOLVE-B data in Figure 7. The baryon fractions peak at a similar group halo mass, although the behavior at low group halo mass appears somewhat flatter and more scattered than observed in the data. The spur seen at high group halo mass and low stellar or cold-baryon fraction is due to a population of galaxies in the SAM with low mass given their brightness, probably due to inadequate consideration of dust. Examination of this galaxy population reveals that they are massive, blue, and star-forming galaxies. To produce the observed galaxy magnitudes, the SAM performs stellar population synthesis modeling based on the star formation and metallicity history of the modeled galaxy, and then applies a physical model for dust as described in Lacey et al. (2016). The dust fraction, extinction curve shape, albedo, and thus, optical depth are all set to the locally measured value of the solar neighborhood, which may not be applicable to galaxies of all masses. The attenuation of starlight by the dust is computed at all wavelengths and then redistributed as a blackbody toward infrared wavelengths. Geometric effects are taken into account by assuming random orientations of the galaxies. The assumptions used and the inherent uncertainties when modeling dust may result in the under-extinction of massive, blue, star-forming galaxies. Recent work by Merson et al. (2016) also shows that these massive blue galaxies are too large, and thus the dust density is too small, leading to inefficient absorption of blue and UV light. The under-attenuation of light in massive galaxies is also apparent in Figure 30 of Lacey et al. (2016), which examines the $g-r$ color distribution of SAM galaxies as a function of brightness compared to SDSS data, finding that in the most massive bin, the SAM shows a bimodal color distribution, whereas the data show only red galaxies. Moreover, we find that performing HAM with group $M_{\text {star }}$ (Figure 11) causes the spur to go away, as the stellar population effects are removed. Group halo masses are then reduced (typically by $\sim 1 \mathrm{dex}$ ) on the $x$-axis, which also causes the stellar and cold baryonic fractions to increase on the $y$-axis. 

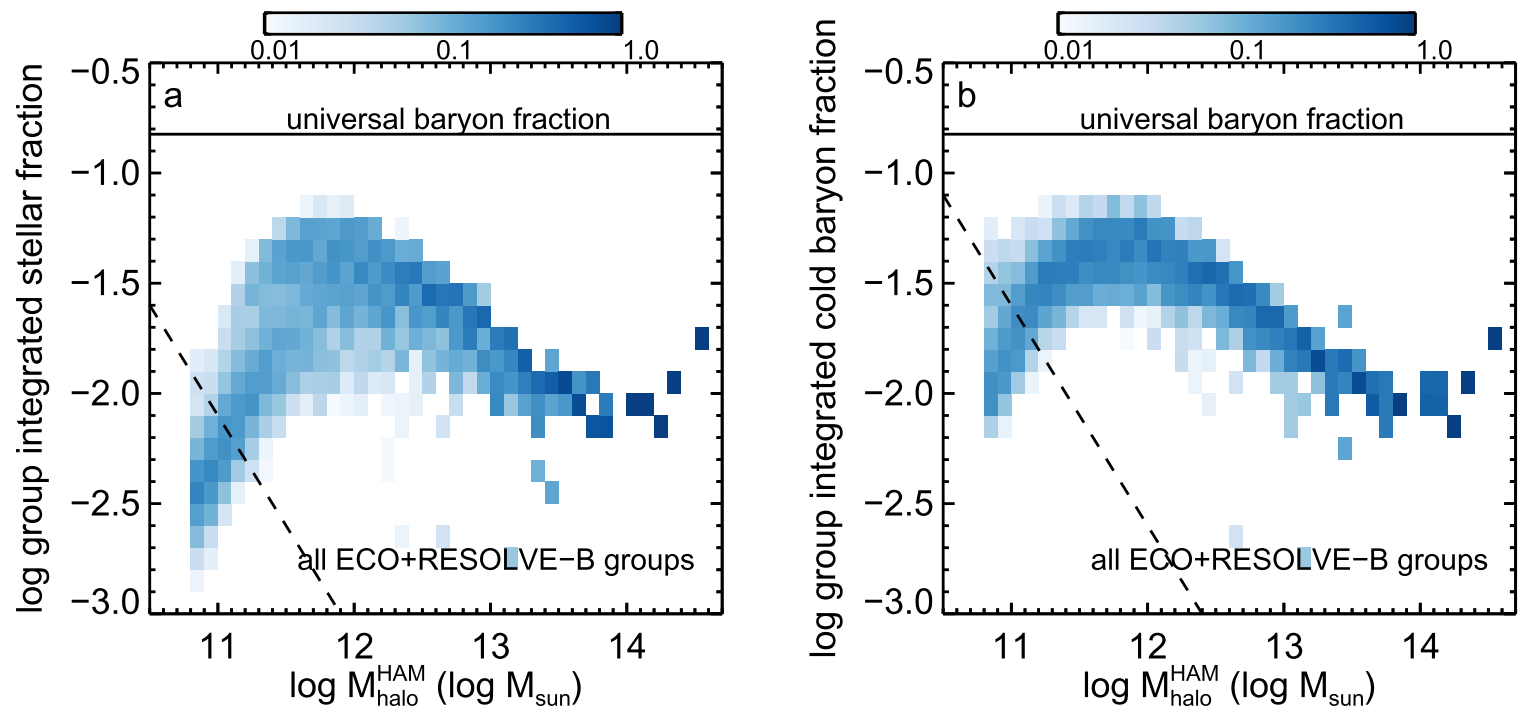

Figure 7. Conditional density plot of group-integrated (a) stellar and (b) cold-baryon fraction as a function of $M_{\text {halo }}^{\text {HAM }}$ based on group $L_{r}$ for all ECO and RESOLVE-B groups. The number densities are weighted by the central completeness corrections described in Section 2.4. The universal baryon fraction (0.15) is shown as a line at the top and the group $M_{\text {star }}$ and $M_{\text {bary }}^{\text {cold }}$ completeness limits are shown by the dashed black lines. The group-integrated stellar mass fraction falls sharply above and below group halo mass $\sim 10^{11.8} M_{\odot}$. The group-integrated cold-baryon fraction reaches a maximum over the nascent group regime $\left(10^{11.4-12} M_{\odot}\right)$ with little scatter. At high group halo mass, the stellar and cold-baryon fractions are similar.

Using the true group halo masses (Figure 12) for the SAM yields completely different results. The SAM cold-baryon fractions vary strongly below a halo mass of $\sim 10^{13} M_{\odot}$ and reach a peak dispersion of over $2 \mathrm{dex}$ at $M_{\text {halo }} \sim 10^{12} M_{\odot}$. This dispersion is much larger than what we find in the data even using the dynamical masses. The SAM suggests that there should be a population of extremely low cold-baryon fraction groups over precisely the same regime where our data suggest groups are reaching a maximum baryonic collapse efficiency.

Analysis of the SAM confirms the serious issue with studying cold-baryon fractions of groups using HAM, which has already been suggested by our dynamical mass analysis: the built-in relationship between group luminosity (or stellar mass) and group halo mass produces the tight relation between the group baryon fraction and group halo mass. There is evidence that groups can have widely varying ratios of hot X-ray gas to collapsed baryons (Roberts et al. 2016), and the HAM algorithm does not account for this diversity, treating all groups of similar luminosity (i.e., similar collapsed baryon content) to be the same. Even with the widely varying coldbaryon fractions in the SAM, using FOF and HAM produces the familiar upside-down U shape seen in all HAM analyses.

To highlight this issue, we show the ratio of hot halo gas to cold (collapsed) gas in galaxies from the SAM mock true groups in Figure 13(a). The SAM hot halo gas includes all gas outside of the galaxy, both halo gas that can be accreted onto galaxies and gas ejected from the galaxy by feedback. The plot shows that low-mass groups and high-mass groups have ratios of hot-to-collapsed gas of around $\sim 10$ and $\sim 100$, respectively, but in the nascent group regime $\left(\sim 10^{11.4-12} M_{\odot}\right)$, the ratio of hot-to-collapsed gas becomes widely varying. It may be that dynamical mass estimates better recover this scatter in hot-tocold baryon fractions for lower-mass groups. We further discuss the wide variation in hot-to-collapsed gas in the SAM and its relation to the implementation of feedback in the SAM in Section 4.1.

Figure 13(a) also shows that low-mass true groups in the SAM all have ratios of hot-to-collapsed gas mass that are much lower than in high mass halos. At the same time, the low-mass observed groups in ECO have low cold-baryon fractions using dynamical masses (Figure 9), which decrease toward lower halo masses. Taking the theoretical and observational results together may seem inconsistent with a constant baryon fraction. Figure 12(b) does appear to be consistent with Figure 9(b) in terms of the observed and theoretical cold-baryon fractions for low-mass systems. The large change in hot-to-cold gas ratios in Figure 13(a) is put into perspective by Figure 13(b), which shows that much of the change is driven by the stellar content of low- and high-mass groups. Furthermore, the SAM's definition of cold gas, computed by determining the amount of cooling gas that has had enough time to reach the center of the halo (thus governed by the cooling and free-fall times), may overestimate the cold gas because it does not include the effect of ionization by internal radiation from star formation within the galaxy. Thus, a small amount of the cold gas in the SAM could actually be ionized gas within the galaxy disk that would not be observable in $\mathrm{HI}$.

\subsection{Adding The Hot Gas}

In large groups, the dominant baryonic component is the hot X-ray-emitting gas (e.g., Mitchell et al. 1977; White et al. 1993; Giodini et al. 2009), thus the large offset between the group CBMF and group HMF at large group halo masses is not unexpected.

To produce a group BMF that includes hot gas mass, we use the hot gas fraction scaling relation given by Giodini et al. (2009), which is calibrated on groups and clusters from the COSMOS survey,

$$
f_{\text {gas }}=9.3\left(\frac{M_{500 c}}{2 \times 10^{14}}\right)^{0.21}
$$

Their calibration, however, was performed for groups with halo masses defined at $M_{500 c}$ (i.e., an overdensity of 500 times the critical density of the universe). To use their calibration, we 

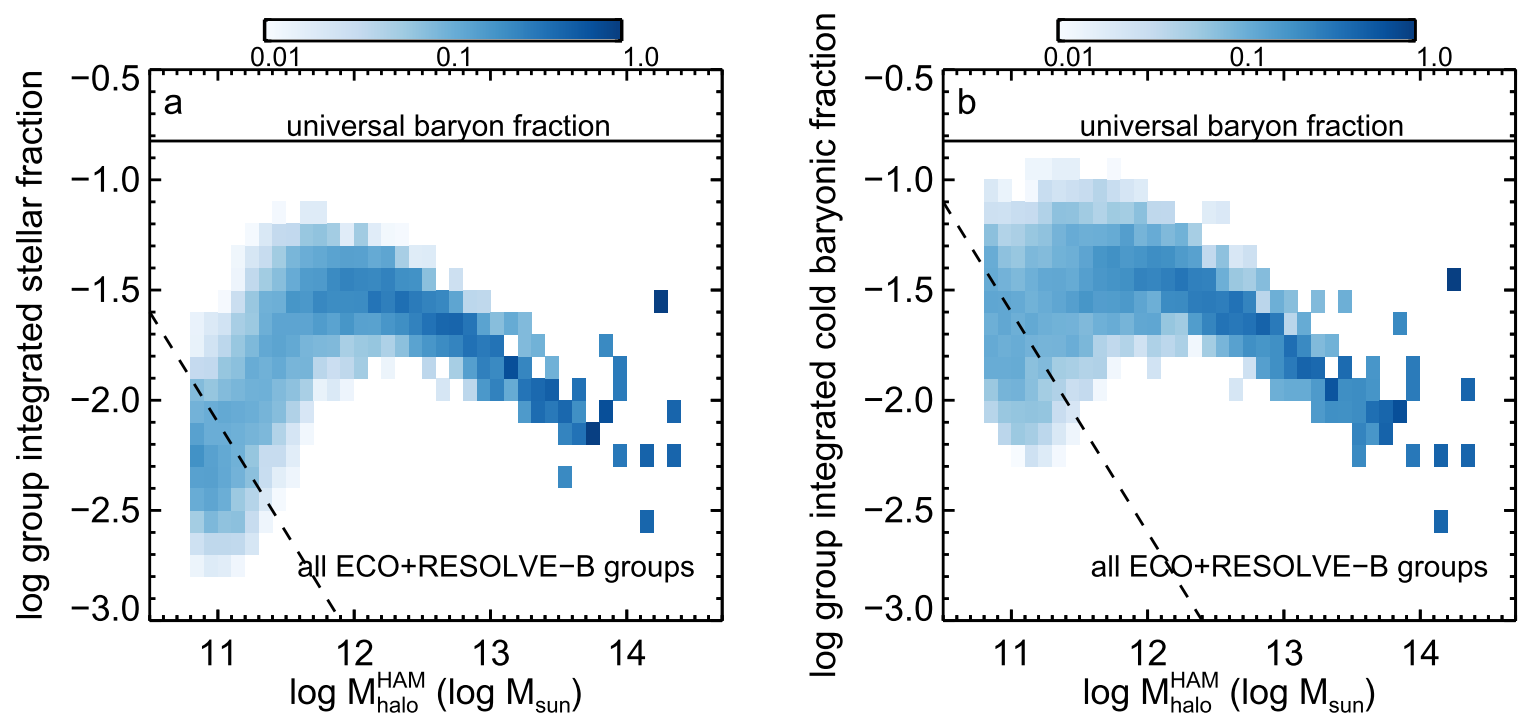

Figure 8. Same as Figure 7, except using $M_{\text {halo }}^{\text {HAM }}$ based on group $M_{\text {star }}$ abundance matching with scatter of 0.14 dex added (to match the scatter based on group luminosity). The group-integrated stellar fractions are similar to those using the group masses matched on $L_{r}$. The group-integrated cold-baryon fractions have increased scatter at low masses compared to the $L_{r}$ version. The increased scatter is likely due to the fact that $L_{r}$ correlates more closely with cold baryonic mass than with stellar mass.

must scale the gas fraction for halo masses defined at $M_{280 b}$. We assume that the dark matter halo mass density follows an NFW profile (Equation (7)) and that the hot gas density distribution follows a beta-model profile (Equation (8)),

$$
\begin{aligned}
& \rho_{\mathrm{dm}}=\frac{\rho_{0, \mathrm{dm}}}{\frac{r}{r_{s}}\left(1+\left(\frac{r}{r_{s}}\right)^{2}\right)}, \\
& \rho_{g}=\rho_{0, g}\left(r^{2}+r_{c}^{2}\right)^{\frac{-3 \beta}{2}} .
\end{aligned}
$$

The scale radius of the NFW profile, $r_{s}$, is determined by the virial radius and halo concentration and $\rho_{0, \mathrm{dm}}$ is the virial overdensity of dark matter. For the hot halo gas profile, $r_{c}$ is the core radius of the profile, $\rho_{0, g}$ is the gas density normalization, and $\beta$ is the power-law slope. Based on the $\beta$ model fits from simulations in Eke et al. (1998), we set $r_{c}=r_{s} / 3$ and $\beta=2 / 3$. We integrate these two density distributions out to $r_{500 c}$, and we use the value of $f_{\text {gas }}$ at $r_{500 c}$ (from Giodini et al. 2009) to determine the ratio of $\rho_{0, \text { gas }}$ to $\rho_{0, n f w}$. Finally, we integrate the two density distributions out to $r_{280 b}$ and determine the new $f_{\text {gas }}$ calibration given in Equation (9),

$$
f_{\mathrm{gas}}=10.3\left(\frac{M_{280 b}}{2 \times 10^{14}}\right)^{0.21} .
$$

In Figure 14, we show the group CBMF (blue) and BMF including hot gas (red) for ECO using the HAM mass estimates to determine the hot gas component. (Results are similar using the dynamical mass estimates.) We compute both the group CBMF and the group BMF using the median group cold baryonic mass measurement for simplicity (using Poisson statistics to compute error bars). Including the hot gas significantly changes the shape of the group BMF at high halo masses, causing it to track the HMF down to group $M_{\text {bary }} \sim 10^{11} M_{\odot}$.

The fact that the group BMF does not line up exactly with the group HMF (by a factor of $\sim 2$ ) at high masses suggests that there is still some missing baryonic component that we have not included. Possibilities include (1) intracluster light that contributes $\sim 10 \%$ (Feldmeier et al. 2004; Krick et al. 2006), (2) low-mass satellite galaxies below our mass limits (contributing at most 14\%; see Section 2.3.1), and (3) warmhot gas, WHIM, too cool to emit in X-rays, which potentially contributes $40 \%-50 \%$ of the baryons based on simulations (Davé et al. 2001; Cen \& Ostriker 2006). We also note that most studies of the hot X-ray gas in clusters find that accounting for the hot X-ray gas and stars does not completely account for all the baryons (Gonzalez et al. 2007; Giodini et al. 2009; Main et al. 2016).

Another consideration is that much of the WHIM may lie outside the virial radius of the halo due to supernova-driven outflows. From simulations of Milky Way galaxies, Sokolowska et al. (2016) find that $20 \%-30 \%$ of the WHIM is pushed out between 1 and 3 virial radii, and that $90 \%$ of the universal baryon fraction is recovered only when considering the halo gas out to 3 virial radii (much further than the considerations used in our analysis). Examining simulations of lower-mass galaxies $\left(M_{\text {halo }} \sim 10^{11} M_{\odot}\right)$, Wang et al. (2017) also find that baryons are expelled beyond twice the virial radius. Such feedback may also cause groups to begin forming with a depleted baryon fraction (Liang et al. 2016). Thus, the expectation that we should be able to account for all of the baryons in groups and clusters to the virial radius may be false, and the shortfall, while interesting, may not necessarily be problematic.

\section{Discussion}

In this section, we present further discussion of the results of this work. First, we consider the relationship between baryonic collapse efficiency and galaxy and group growth, examining in particular the nascent group regime. Second, we discuss possible undetected baryons in galaxies and their halos and consider the effects of these mass components on the group BMF. 

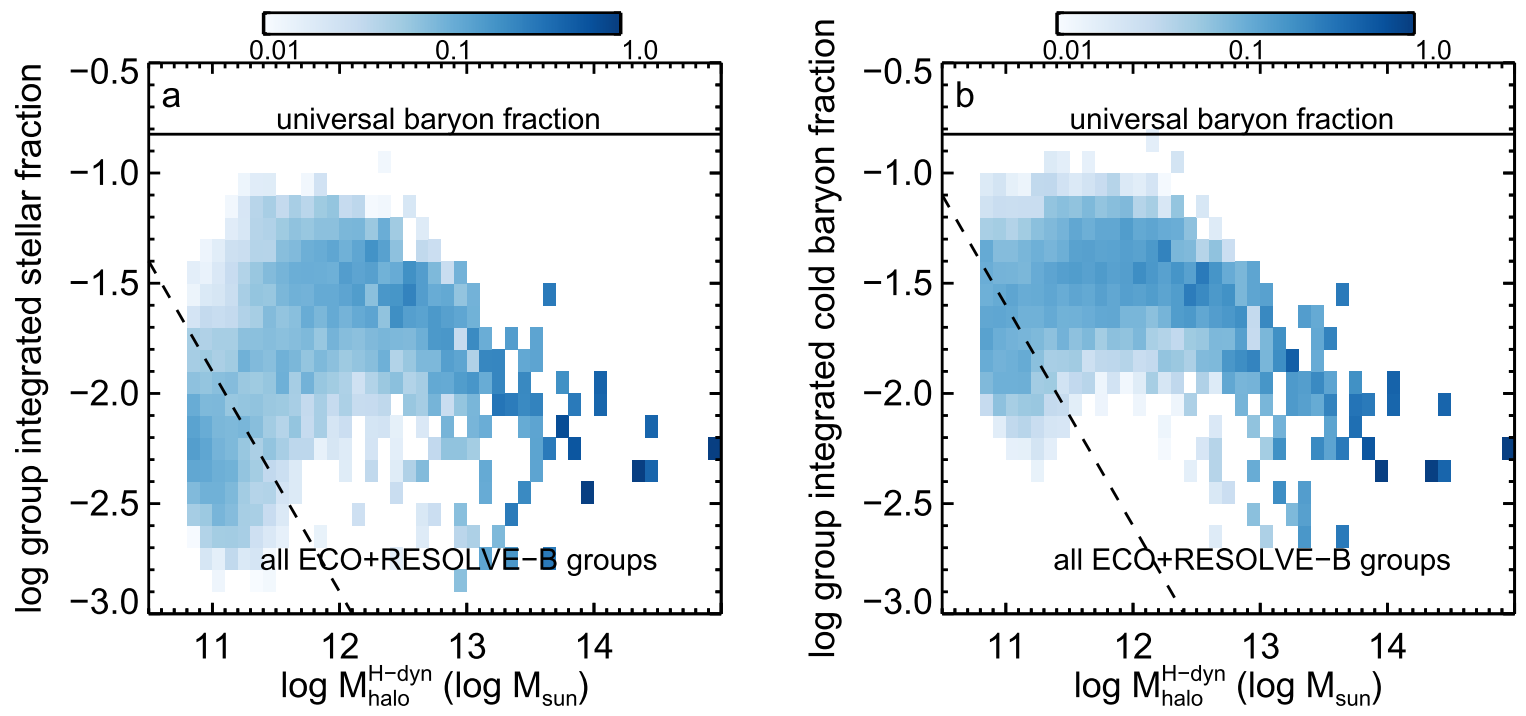

Figure 9. Conditional density plot of the group-integrated (a) stellar and (b) cold-baryon fractions as a function of $M_{\text {halo }}^{\text {H-dyn }}$ determined combining the $N \geqslant 3$ dynamical and low- $N$ HAM estimates for both ECO and RESOLVE-B groups combined. The universal baryon fraction is shown as a line at the top and the group cold baryonic mass completeness limit is shown by the dashed black line. Using the dynamical mass results in a wider range of group stellar and cold-baryon fractions, particularly over the nascent group halo mass range (and below, but here the scatter is introduced; see Section 2.3.5). The peak baryonic collapse efficiency occurs near $\sim 10^{12} M_{\odot}$.

\subsection{Baryonic Collapse Efficiency and Galaxy Growth: From Isolated Dwarfs to Nascent Groups}

At the lowest group halo masses $\left(<10^{11.4} M_{\odot}\right)$, we have seen that groups are mostly isolated dwarf galaxies in $N=1$ halos and are increasing their cold baryonic mass faster than their dark matter halo mass (Figures 7-9). Although isolated dwarfs are often thought of as inefficient star formers (due to their large $\mathrm{H}$ I reservoirs, which lead to long gas depletion times), K13 showed that they are nonetheless growing rapidly using a long-term measure of galaxy growth called fractional stellar mass growth rate (FSMGR), defined as the stellar mass produced in the last Gyr divided by the stellar mass produced prior to that Gyr. Isolated dwarf galaxies have FSMGR $\sim 1$, implying stellar mass doubling on Gyr timescales. Additionally, Moster et al. (2013) show that isolated dwarf galaxies in low-mass halos are growing much more rapidly than their halos at current times using multi-epoch abundance matching. Thus, while not at the peak of cold-baryon fraction, such low-mass groups are at peak galaxy growth rates.

Over the halo mass regime of $10^{11.4}-10^{12} M_{\odot}$, the nascent group regime, we find that groups reach peak collapsed baryon fraction or "baryonic collapse efficiency." Here we use "efficiency" in the usual convention as a level reached rather than a rate of processing (e.g., Conroy \& Wechsler 2009; Leauthaud et al. 2012b). As baryonic collapse efficiency peaks, galaxy formation slows and group processes begin to shape the population. Indeed, K13 found that central galaxies in the nascent group regime (using the central galaxy mass-group mass relationship) had lower FSMGR than isolated dwarfs, implying slowed galaxy growth at these group mass scales. Additionally, from examination of the galaxy mass functions in E16, we find that nascent groups may already experience merging and/or stripping of satellites, as the satellite mass function is depressed relative to the central mass function and has a flat low-mass slope (to our completeness limits). Once groups reach this nascent group scale, group processes such as merging and stripping seem to act to stop the growth of the collapsed baryonic content of groups. Group cold-baryon fractions then drop toward higher group halo masses, as the uncollapsed hot halo gas dominates the baryonic content of the halo. Future planned studies of FSMGR as a group-integrated quantity and as a function of group mass may help shed more light on the connection between galaxy growth and group formation.

Connecting these results with the group CBMF (Figure 5), we find that low-mass groups (with peak galaxy formation rates) have group $M_{\text {bary }}^{\text {cold }}<10^{10} M_{\odot}$ (from Figure 1(a)), and thus lie on the shallow power-law slope of the group CBMF. Nascent groups have group $M_{\text {bary }}^{\text {cold }}$ ranging from $\sim 10^{10}-10^{10.8} M_{\odot}$, which places them just below the knee of the group CBMF. The highest-mass halos then lie on the steep falloff toward higher masses (although accounting for their hot gas the falloff has a slope similar to the HMF; Figure 14). That the nascent groups exist right at this change in mass function behavior reflects the fundamental change in baryonic collapse efficiency between lowand high-mass group halos.

We have shown that HAM halo mass estimates yield a tight maximum in the baryonic collapse efficiency, but that dynamical halo mass estimates suggest more scatter. The scatter in the collapsed baryon fraction using dynamical masses is still much smaller than suggested by the SAM true groups. The discrepancy may be partially due to the reliance on HAM for $N=1$ and 2 groups, for which we have no other proxy for the halo mass. The SAM, in addition, may have overly large feedback (as discussed below), causing unrealistic scatter in cold-baryon fractions. To fully discriminate between these two possibilities, we require independent measurements of the halo masses of $N=1$ and 2 groups. The RESOLVE survey is conducting a census of velocity measurements (either resolved rotation curves or velocity dispersions, depending on galaxy type), which can serve as a proxy for $N=1$ group halo masses. Using these measurements, we will in the future attempt to determine how large the scatter in cold-baryon fraction is at low masses and whether it can be attributed to the growing diversity in the ratio of hot halo gas to cold collapsed baryons.

In the SAM, the transition from isolated dwarfs to larger groups over the nascent group regime is strongly governed by 

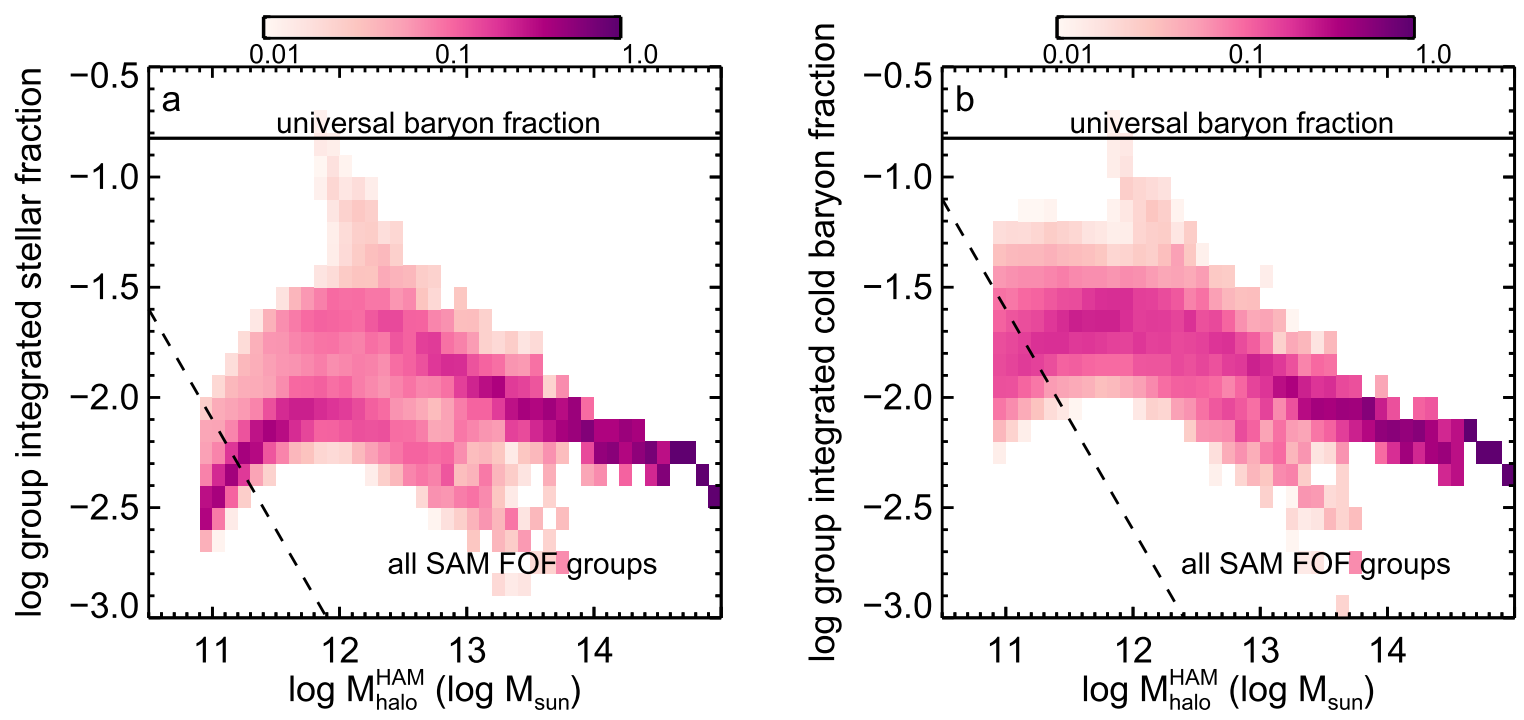

Figure 10. Conditional density plot of group-integrated (a) stellar and (b) cold-baryon fractions for the SAM using the FOF groups with HAM based on group luminosity. We find that applying FOF and HAM to the SAM mock catalog results in the upside-down U shape seen in the ECO data. The spur of the low cold-baryon fraction groups for their halo mass is due to a population of galaxies with much lower masses than their luminosities would suggest. These are large, blue, star-forming galaxies for which the dust correction does not sufficiently extinct their intrinsic magnitudes (see discussion in Gonzalez-Perez et al. submitted).

the model's implementation of feedback. The nascent group regime is where the dominant mode of feedback transitions from stellar feedback (at lower halo masses) to AGN feedback (at higher halo masses). The location of the knee at higher masses in the SAM (and the resulting underprediction of groups in the SAM near the knee of ECO's group SMF and CBMF, Figure 6) reveals that the model's implementation of feedback is overly efficient at these scales. Recently, Mitchell et al. (2016) compared the GALFORM galaxy SMF with observations, finding that the location and amplitude of the knee of the galaxy SMF are very sensitive to the feedback prescription in the SAM and that reducing the stellar feedback efficiency improves agreement in the knee with observed galaxy SMFs.

Additionally, the SAM true groups exhibit extremely varied cold-baryon fractions and hot-to-collapsed gas ratios over the nascent group regime (see Figures 12 and 13). This scatter could be partially driven by the transition from stellar to AGN feedback, which occurs over the nascent group regime as galaxies transition to the group environment. Some of the variation may also be a result of the stellar feedback implementation in GALFORM, which ties the fraction of cold gas ejected from the disk (the mass-loading factor) to the circular velocity of the galaxy disk or bulge for quiescent or starburst star formation episodes, respectively. Comparing the central galaxy stellar to halo mass (SHM) relationship from GALFORM and L-GALAXIES, a different SAM described in Guo et al. (2011) that ties the mass-loading factor to the halo circular velocity, Guo et al. (2016) find a larger scatter in the SHM relationship of GALFORM than that of L-GALAXIES. Additionally, Mitchell et al. (2016) find that implementing the L-GALAXIES mass-loading factor prescription in GALFORM reduces the scatter in the SHM relationship. Thus, accurately quantifying the scatter in cold-baryon fractions (or the SHM relationship) over nascent group scales can provide important constraints that lead to improved models of galaxy formation.

\subsection{Undetected Gas}

So far, we have only considered the effect of hot X-ray gas that dominates the mass of large groups and clusters. There are other gas components, however, that may especially affect galaxy and group mass, such as opaque $\mathrm{HI}$ gas, CO-traced molecular gas, CO-dark molecular gas, and WHIM. We have not taken into account $\mathrm{H}$ I self-absorption (opaque $\mathrm{HI}$ ), which could contribute up to $30 \%$ of the H I gas in the most edge-on galaxies (Giovanelli et al. 1994). We also neglected the COtraced molecular gas in this work, under the assumption that it rarely dominates the cold gas in galaxies. This assumption is not true for some large spiral galaxies, but those are generally dominated by their stellar mass (K13; Boselli et al. 2014).

In low-mass, low-metallicity, gas-rich galaxies, molecular gas is difficult to detect with standard tracers like $\mathrm{CO}$ (Taylor et al. 1998; Leroy et al. 2005; Schruba et al. 2012; Bolatto et al. 2013) due to the low dust content, which allows the CO to be photodissociated more easily. The molecular hydrogen, on the other hand, is self-shielded, making it traceable by the [CI] and [CII] lines (Röllig et al. 2006; Glover et al. 2015). Ongoing work using the Herschel Dwarf Galaxy Survey seeks to uncover CO-dark gas through examination of these far-IR lines, potentially finding between ten and several hundred times as much CO-dark as CO-traced molecular hydrogen (Madden et al. 2016). Although the CO-dark molecular gas is mainly thought to contribute significantly to the gas mass of lowmetallicity dwarfs, Pineda et al. (2013) find that $~ 30 \%$ of the molecular gas mass in the Milky Way could be CO-dark molecular gas. In fact, the theoretical model of Wolfire et al. (2010) predicts a CO-dark gas fraction of 0.3 for Milky-Waylike extinction, and an increasing CO-dark gas fraction for decreasing extinction values, which may be more applicable to low-metallicity dwarf galaxies.

Another contributor to the undetected gas component is the WHIM. Based on simulations, WHIM gas in the galaxy halo contributes significantly to the baryon census ( 40\%-50\%; Cen \& Ostriker 2006; Smith et al. 2011), although a significant amount of WHIM may be pushed outside the virial radius in 

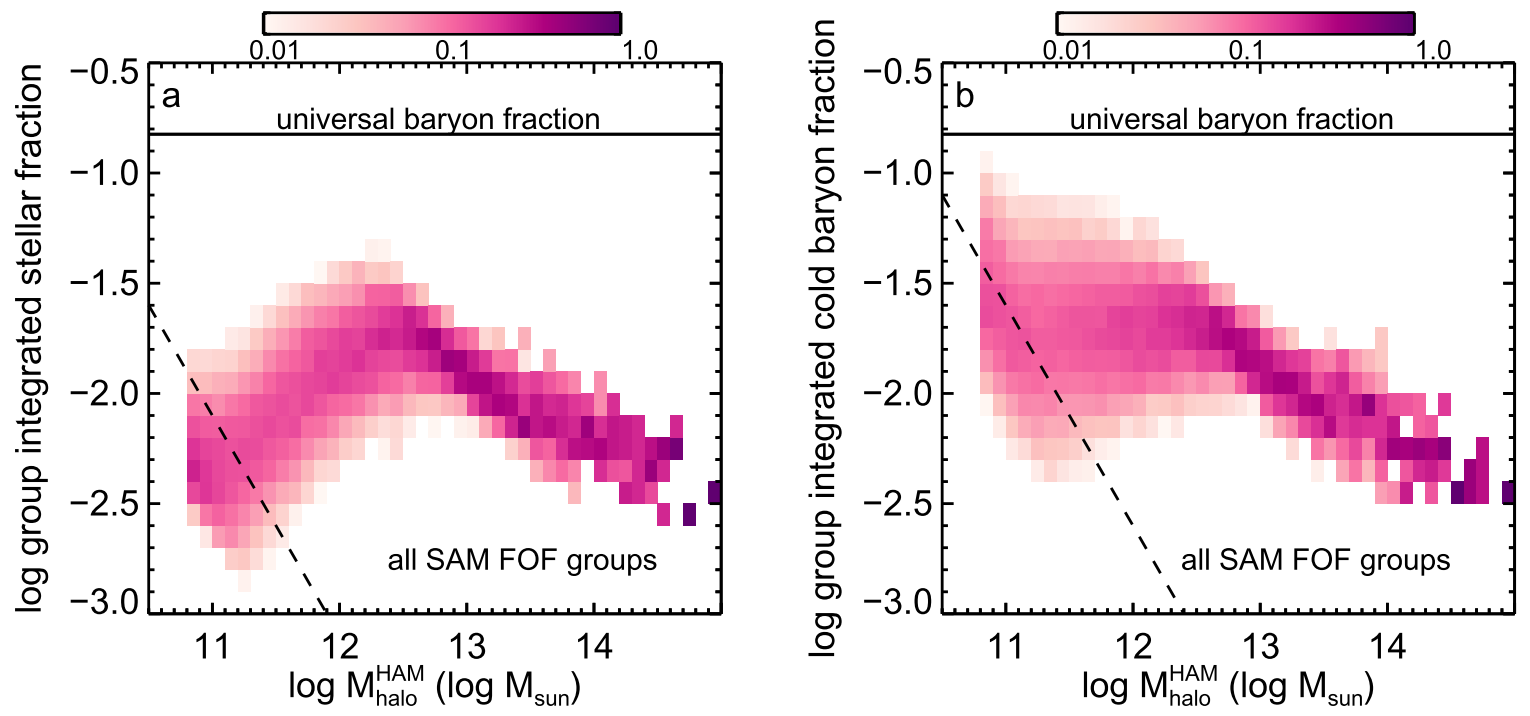

Figure 11. Same as Figure 10 but using HAM based on group-integrated stellar mass, which removes the low cold-baryon fraction spur.

Milky-Way-size halos (Sokołowska et al. 2016). Observations of X-ray absorption lines have yielded large variations in the mass and distribution of the WHIM in the Milky Way: from $\sim 4 \times 10^{8} M_{\odot}$ within $20 \mathrm{kpc}$ (Bregman \& Lloyd-Davies 2007) to $\sim 10^{10} M_{\odot}$ within $100 \mathrm{kpc}$ (Gupta et al. 2012).

Studies of the baryonic Tully-Fisher relation suggest that there could be missing gas in the galaxy disk that scales with the H I gas component (Pfenniger \& Revaz 2005; Begum et al. 2008; Revaz et al. 2009). These studies find that a multiplicative factor of 3-11 applied to the $\mathrm{H}$ I mass produces a tighter Tully-Fisher relation across dwarf to giant galaxy scales. Additional indirect evidence for such undetected gas is also seen in rotation curve decomposition analyses (Hoekstra et al. 2001; Swaters et al. 2012), which find that a direct scaling of the H I gas or baryonic distribution can explain the galaxy rotation curve. Although the above-described potential forms for the undetected gas may not explain such large multiples of the H I mass, they do all point to a missing reservoir that could contribute significantly to the galaxy mass.

To examine the effect of including such undetected gas in our group mass functions, we first note that scaling the HMF by a factor of 0.07 (the universal baryon fraction 0.15 divided by 2; dashed black line in Figure 15) yields agreement between the HMF and the group BMF that includes hot gas (red histogram) down to group $M_{\text {bary }} \sim 10^{10.7} M_{\odot}$. This factor of 2 reduction is in rough agreement with expectations that the WHIM contributes $40 \%-50 \%$ of the baryons in group halos. Figure 15 also shows the effect of including undetected galaxy gas that scales with the galaxy H I gas component (green histogram). We only scaled the cold baryonic masses of lowmass $\left(M_{\text {star }}<10^{9.5} \quad M_{\odot}\right)$, gas-rich $\left(1.4 M_{\mathrm{H}} / M_{\mathrm{star}}>1.0\right)$ galaxies, which are the most likely to harbor a significant amount of undetected CO-dark gas. We multiply their cold H I gas mass by a factor of 2 . Such scaling affects the low-mass end of the group BMF and improves agreement with the scaled HMF down to $M_{\text {bary }} \sim 10^{10.3} M_{\odot}$. In these analyses, as for our hot gas analysis, we also used the median stellar and gas mass (baryonic-stellar) measurements rather than the full likelihood distribution for simplicity.

Above $10^{11} M_{\odot}$, there is not much change in the total group $\mathrm{BMF}$, since there are few low-mass, gas-rich galaxies in highmass halos. Below $10^{11} M_{\odot}$, the group BMF including hot gas and this scaled cold gas component continues to track the scaled HMF better, lending support to the idea that undetected gas may contribute significantly to the galaxy component of the group mass, particularly at nascent group scales and below.

\section{Conclusions}

In this work, we examined the group-integrated stellar and baryonic contents of groups for the RESOLVE and ECO surveys. We further compared with results from an SAM mock catalog and discussed implications for group formation, particularly in the nascent group regime.

1. The group SMF and CBMF exhibit steep slopes at high masses, and a shallower rising slope at low masses. They most closely approach the dark matter HMF near $\sim 10^{11} M_{\odot}$. The low-mass slope of the group CBMF is steeper than that of the group SMF, but still deviates from the steep dark matter HMF slope (see Section 3.1 and Figure 5).

2. The SAM's group SMF and CBMF are similar to those of ECO at high masses. However, the SAM has fewer groups at the transition mass of $\sim 10^{11} M_{\odot}$, and the SAM mass functions rise more steeply at low masses (Figure 6). These differences are likely due to the sensitivity to the transition between stellar and AGN feedback in the models (Section 4.1).

3. Inclusion of hot halo gas in the group BMF using a literature prescription produces a slope that runs parallel to the dark matter HMF (although still low by a factor of 2; Figure 14). The hot halo gas does not contribute significantly to the group baryonic mass below $\sim 10^{11} M_{\odot}$ (see Section 3.3).

4. If we assume that there is additional undetectable gas in galaxies that scales with the $\mathrm{HI}$ mass for low-mass, gasrich galaxies (which may have large reservoirs of COdark molecular gas), by adopting a multiplicative factor of 2 , as has been suggested by baryonic Tully-Fisher studies, we can produce a more steeply rising low-mass slope below $10^{11} M_{\odot}$ that continues to run parallel to the dark matter HMF (Section 4.2 and Figure 15).

5. Examination of the stellar and cold-baryon fractions as a function of HAM group halo mass reveals the familiar upside-down U shape seen in previous work (Figures 7 

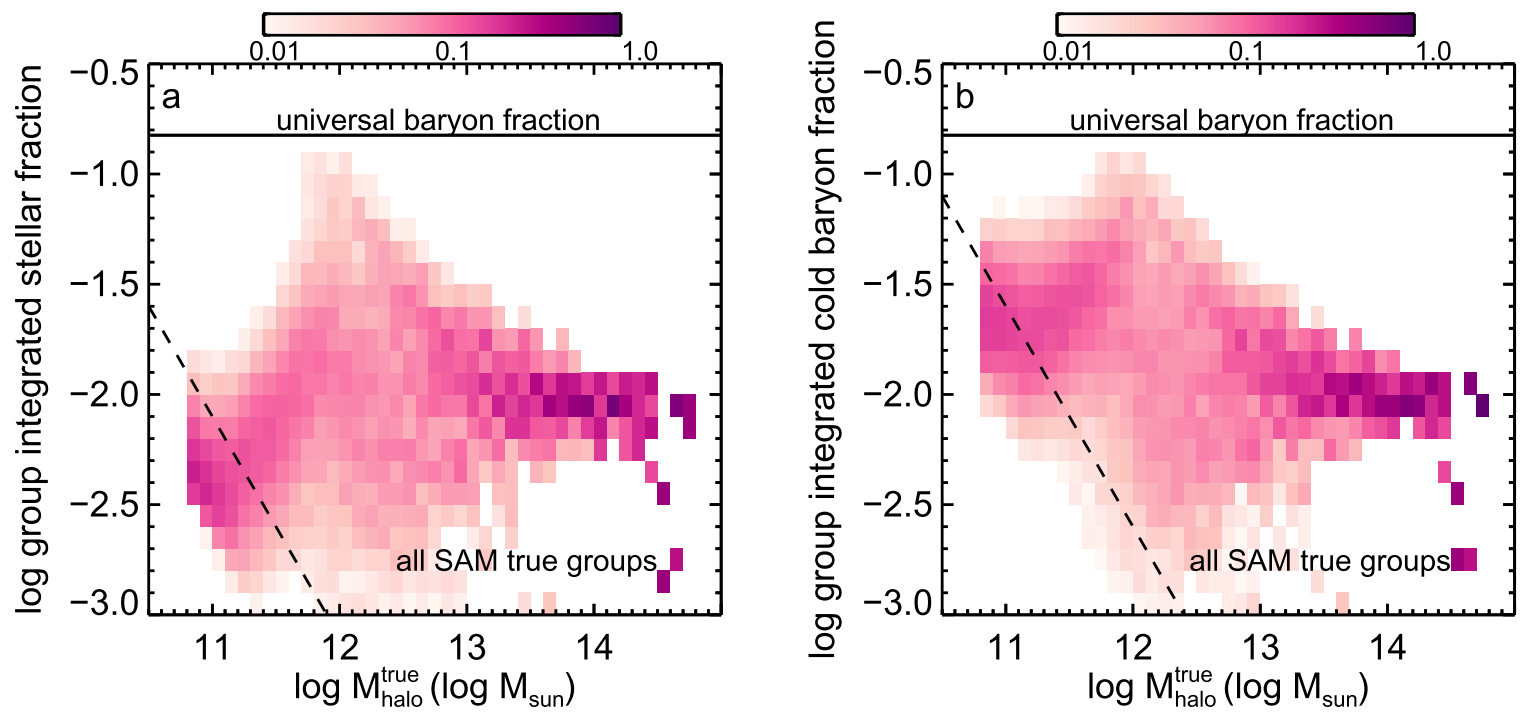

Figure 12. Conditional density plot of group-integrated (a) stellar and (b) cold-baryon fractions for the SAM using the true group halo mass. We find that below $\sim 10^{13} M_{\odot}$, the stellar and cold-baryon fractions become extremely varied, even more so than seen with the dynamical mass estimates for ECO and RESOLVE-B.

and 8). Although the group stellar fraction has a narrow peak near $\sim 10^{11.8} M_{\odot}$, the peak of the group cold-baryon fraction (or baryonic collapse efficiency) is spread across a broader halo mass range of $\sim 10^{11.4}-10^{12} M_{\odot}$. This broad peak of the baryonic collapse efficiency coincides with the nascent group regime, wherein galaxies and galaxy groups transition from gas rich to stellar dominated.

6. Because HAM halo masses enforce monotonicity between group halo mass and collapsed baryons as quantified by group $L_{r}$ or $M_{\text {star, }}$, but we are interested in the diversity of collapsed baryon to halo mass ratios, we developed a new way of measuring dynamical masses that allows us to probe halo mass independent of collapsed baryon properties. Our hybrid halo masses smoothly transition from using HAM for $N=1$ and 2 groups, stacked dynamical masses for $N>2$ groups, and individual group dynamical masses for the highest $N$ groups.

7. Examination of the stellar and cold-baryon fractions as a function of dynamical halo mass estimates suggests more scatter in collapsed baryon fractions than observed using HAM halo mass estimates (Figure 9), potentially reflecting variations in the hot-to-collapsed baryon fraction between groups at fixed group mass. This result argues for caution in interpreting baryon fractions using HAM, as the built-in assumption of mass following collapsed baryons may break down across intermediate group halo mass regimes.

8. The SAM true groups also suggest that there should be a population of very low cold-baryon fraction groups (Figure 12). Once HAM is performed on the SAM, we obtain the same upside-down $U$ shape as seen in the data (Figures 10 and 11). This result underscores the importance of recognizing the built-in relationship between halo mass and group cold baryonic mass when using HAM halo mass estimates.

The results from this paper touch on several aspects of the baryon census. For example, we showed that the group BMF can obtain a similar shape to the HMF once the collapsed baryonic matter within groups is combined with the hot halo gas and potential CO-dark gas in gas-rich, dwarf galaxies. Although the group BMF is shifted lower in mass by a factor of $\sim 2$, that shift is in agreement with WHIM estimates of $40 \%-$ $50 \%$, and this result suggests that at the scales we probe $\left(M_{\text {halo }} \sim 10^{11-14.5} M_{\odot}\right)$, we can account for most of the baryons. Using dynamical masses to explore group cold-baryon fractions, however, points to far more variation in the hot-tocollapsed baryon ratio in groups than implied by using HAM, especially across the nascent group regime. The SAM provides additional support for large variations in the hot-to-collapsed baryon fraction at nascent group scales. Nascent groups appear to be sites of active group formation processes such as merging and stripping, as shown by the depressed, flat low-mass slope of the nascent group galaxy mass function in E16. The nascent group regime, however, is where our stacked dynamical mass analysis starts to break down as we approach the acutely low- $N$ regime, thus leaving us unable to fully probe these variations. In future work, we plan to measure dynamical masses using internal galaxy kinematics to extend our analysis to the lowest$N$ groups, enabling combined analysis of the galaxy and group (subhalo and halo) velocity functions.

We thank the referee for the comments, which have improved this work. We thank Matt Bayliss for giving us the idea of stacking groups to estimate dynamical masses at low $N$. We also thank Brian McNamara for useful discussions of group baryon fractions. We would like to thank Prajwal Kafle and Laura Parker for helpful conversations regarding group dynamical mass estimation and virialization status. We are grateful to Maud Galametz and Aleksandra Sokolowska for discussions of COdark molecular gas in galaxies and ionized gas in halos, respectively. We also thank Peter Mitchell for discussions of feedback related to the knee of the group mass function. K.D.E. and S.J.K. acknowledge support for this research from NSF grant AST-0955368. K.D.E. also acknowledges support from NSF grant OCI-1156614, the North Carolina Space Grant Fellowship, the University of North Carolina Royster Society of Fellows, and the Sigma Xi Grants-in-Aid of Research Program. This work is based on observations from the SDSS. Funding for SDSS-III has 

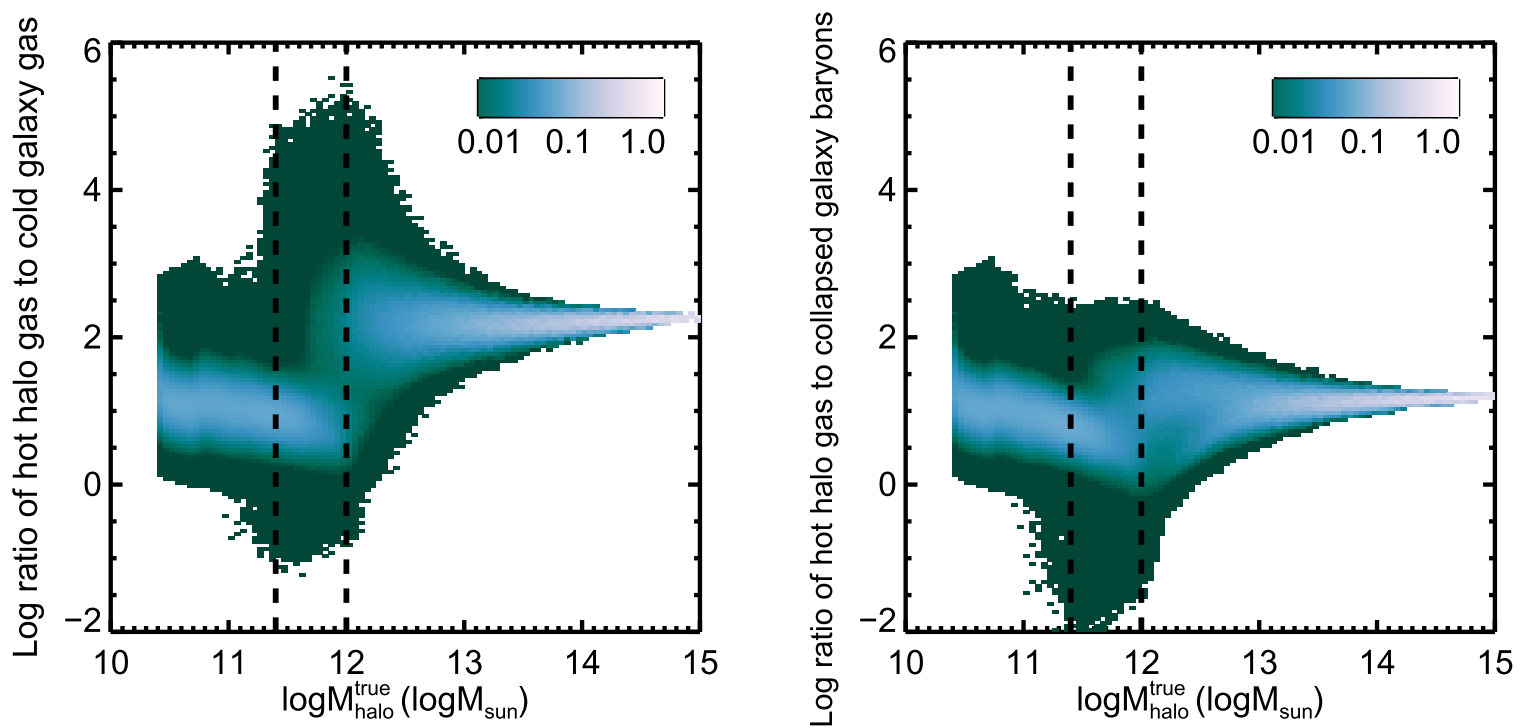

Figure 13. Conditional density plot of the ratio of hot halo gas to (a) cold (collapsed) gas mass and (b) cold (collapsed) baryonic mass in galaxies for the SAM true groups. Over the nascent group halo mass regime, the scatter in ratio between the hot halo gas to collapsed galaxy gas increases as galaxies transition from single objects within their halo to larger groups. The overall change in hot-to-cold gas fraction between low- and high-mass groups is driven by their differing stellar content, as revealed in panel (b), which includes the cold gas and stellar content of groups in the denominator.

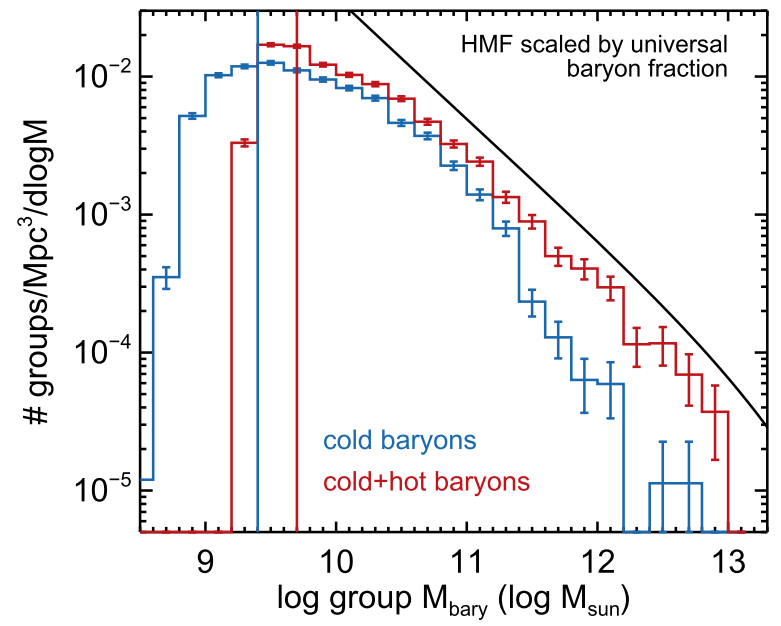

Figure 14. ECO group CBMF (blue) and BMF including hot X-ray gas (red), where we used the hot gas prescription based on the dark matter halo mass from Giodini et al. (2009), scaled for our halo mass definition. The completeness limits are shown by the vertical lines corresponding to the colors of the histograms. The BMF completeness limit is determined by finding the maximum hot gas correction to group $M_{\text {bary }}^{\text {cold }}$ at $10^{9.4} M_{\odot}$, which we find to be $\sim 0.3$ dex. Including the hot gas causes the group BMF to run parallel to the dark matter HMF. In this analysis, for simplicity, we do not use full group mass likelihood distributions but instead use the median values. Error bars represent the Poisson statistics.

been provided by the Alfred P. Sloan Foundation, the Participating Institutions, the National Science Foundation, and the US Department of Energy Office of Science. The SDSS-III Web site is http://www.sdss3.org/. SDSS-III is managed by the Astrophysical Research Consortium for the Participating Institutions of the SDSS-III Collaboration including the University of Arizona, the Brazilian Participation Group, Brookhaven National Laboratory, Carnegie Mellon University, University of Florida, the French Participation Group, the German Participation Group, Harvard University, the Instituto de Astrofisica de Canarias, the Michigan State/Notre Dame/JINA Participation Group, Johns Hopkins University, Lawrence Berkeley National Laboratory, Max Planck Institute for Astrophysics, Max Planck Institute for

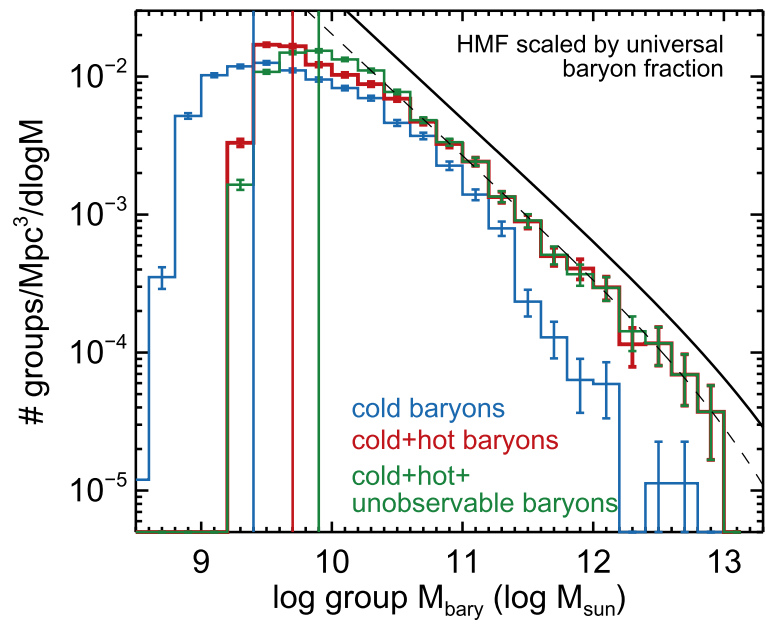

Figure 15. Group CBMF for ECO (blue), BMF including hot gas (red, based on HAM), and BMF including both hot gas and potentially undetected gas in low-mass, gas-rich galaxies (green). We find agreement between the BMF including hot gas and the HMF rescaled by a 0.07 (a factor of 2 smaller than the universal baryon fraction; dashed black line) down to $M_{\text {bary }}=10^{10.7} M_{\odot}$. By scaling low-mass, gas-rich galaxies, which may harbor large undetected gas components, we extend this agreement to $M_{\text {bary }}=10^{10.3} M_{\odot}$.

Extraterrestrial Physics, New Mexico State University, New York University, Ohio State University, Pennsylvania State University, University of Portsmouth, Princeton University, the Spanish Participation Group, University of Tokyo, University of Utah, Vanderbilt University, University of Virginia, University of Washington, and Yale University. This work is based on observations made with the NASA Galaxy Evolution Explorer $(G A L E X)$. GALEXis operated for NASA by the California Institute of Technology under NASA contract NAS5-98034. This publication makes use of data products from the Two Micron All Sky Survey, which is a joint project of the University of Massachusetts and the Infrared Processing and Analysis Center/California Institute of Technology, funded by the National Aeronautics and Space Administration and the National Science Foundation. This work is based in part on data obtained 


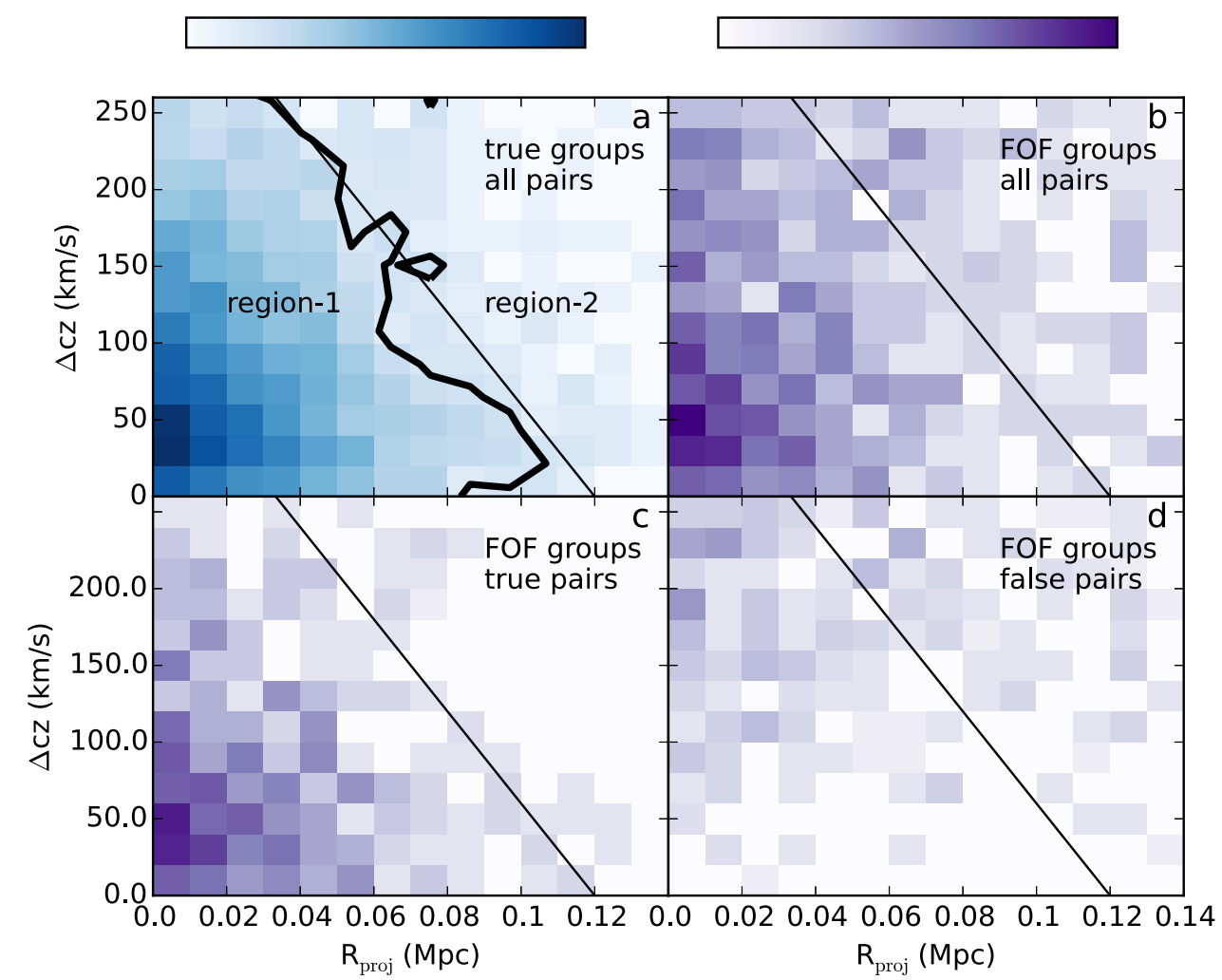

Figure 16. Mock catalog pair statistics, shown as $2 \mathrm{D}$ histograms of the distribution of pairs ( $N=2$ groups) over $\Delta c z$ vs. $R_{\text {proj }}$ space for (a) all true pairs, (b) all FOF pairs, (c) true FOF pairs, and (d) false FOF pairs. (Split-off FOF pairs are not shown, but are distributed roughly equally over this space.) The 2D histograms for panels (b)-(d) are normalized to all FOF pairs, with the color bar shown above panel (b). In panel (a), we show the contour containing 95\% of the true-mock pairs (thick black line), as well as the line we choose to define region- 1 and region-2 (thin black line). Only $5 \%$ of true FOF pairs live in region-2, while nearly $50 \%$ of false FOF pairs live in region-2. We therefore break up all pairs in region-2, improving the percentage of true pairs among FOF pairs from $62 \%$ to $73 \%$.

as part of the UKIRT Infrared Deep Sky Survey. This work uses data from the Arecibo observatory. The Arecibo Observatory is operated by SRI International under a cooperative agreement with the National Science Foundation (AST-1100968), and in alliance with Ana G. Méndez-Universidad Metropolitana, and the Universities Space Research Association. This work is based on observations using the Green Bank Telescope. The National Radio Astronomy Observatory is a facility of the National Science Foundation operated under cooperative agreement by Associated Universities, Inc.

\section{Appendix}

In this appendix, we describe the procedure used to identify and break up false pairs of galaxies in the ECO, RESOLVE-B, and SAM FOF group catalogs. We use a mock catalog built on a $\Lambda$ CDM $N$-body simulation, in which central and satellite galaxies have been placed into halos according to the HOD framework of Berlind \& Weinberg (2002) and redshift space distortions have been included (see M15 for more details).

With this mock catalog, we know the true identities of group halos, and we run the FOF group-finding algorithm to examine how it affects group assignments. Comparing the true and FOF groups, we find that the pair population is often affected by group-finding errors. A large portion of FOF pairs are "false pairs," or two $N=1$ galaxies merged together. We additionally find "split-off" FOF pairs that were originally two members of a larger true group and were split off in the FOF group-finding process. Matching between the true and FOF groups, we identify among FOF groups true pairs, false pairs, and split-off pairs, finding that they comprise $62 \%, 22 \%$, and $16 \%$ of the FOF pair population, respectively.

To break up the false pairs, we examine the distribution of true pairs in $\Delta c z-R_{\text {proj }}$ space, where $\Delta c z$ is the difference in redshift between the pair galaxies and $R_{\text {proj }}$ is computed for the pair as described in Section 2.3.3. The 2D histogram of true pairs is shown in Figure 16(a). We then find the contour that encloses $\sim 95 \%$ of the true pairs (bold black line). To simplify our region, we draw a line reflecting that contour and designate "region-1, " which contains 95\% of true pairs, and "region-2," which contains the remaining $5 \%$ of true pairs.

In panels (b)-(d) of Figure 16, we show the 2D histograms of the overall, true, and false pairs among the FOF groups (normalized to the overall FOF pair population). (Split-off pairs are distributed roughly evenly over the $\Delta c z-R_{\text {proj }}$ space.) In the overall FOF pair population, $5 \%, 48 \%$, and $40 \%$ of the true, false, and split-off pairs reside within region-2. Based on this analysis, we split up all FOF pairs residing in region-2, thereby removing $\sim 1 / 2$ of the false pairs at the expense of splitting up 5\% of the true pairs. (Split-off pairs in region-2 also now contribute to the FOF $N=1$ population, however, they make up $<2 \%$ of the FOF $N=1$ population.) Our new FOF pair population now consists of $73 \%$ true pairs, $15 \%$ false pairs, and $12 \%$ split-off pairs.

\section{ORCID iDs}

Kathleen D. Eckert (1) https://orcid.org/0000-0002-1407-4700 Andreas A. Berlind (1) https://orcid.org/0000-0002-1814-2002 David V. Stark (i) https://orcid.org/0000-0002-3746-2853 


\section{References}

Aihara, H., Allende Prieto, C., An, D., et al. 2011, ApJS, 193, 29

Anglés-Alcázar, D., Faucher-Giguère, C.-A., Kereš, D., et al. 2017, MNRAS, 471, 4693

Balogh, M. L., Mazzotta, P., Bower, R. G., et al. 2011, MNRAS, 412, 947

Beers, T. C., Flynn, K., \& Gebhardt, K. 1990, AJ, 100, 32

Begum, A., Chengalur, J. N., Karachentsev, I. D., \& Sharina, M. E. 2008, MNRAS, 386, 138

Behroozi, P. S., Wechsler, R. H., \& Conroy, C. 2013, ApJ, 770, 57

Berlind, A. A., Frieman, J., Weinberg, D. H., et al. 2006, ApJS, 167, 1

Berlind, A. A., \& Weinberg, D. H. 2002, ApJ, 575, 587

Blanton, M. R., Kazin, E., Muna, D., Weaver, B. A., \& Price-Whelan, A. 2011, AJ, 142, 31

Blanton, M. R., Lupton, R. H., Schlegel, D. J., et al. 2005a, ApJ, 631, 208

Blanton, M. R., Schlegel, D. J., Strauss, M. A., et al. 2005b, AJ, 129, 2562

Bolatto, A. D., Wolfire, M., \& Leroy, A. K. 2013, ARA\&A, 51, 207

Boselli, A., Cortese, L., Boquien, M., et al. 2014, A\&A, 564, A66

Bregman, J. N., \& Lloyd-Davies, E. J. 2007, ApJ, 669, 990

Brown, T., Catinella, B., Cortese, L., et al. 2015, MNRAS, 452, 2479

Calzetti, D. 2001, PASP, 113, 1449

Casoli, F., Sauty, S., Gerin, M., et al. 1998, A\&A, 331, 451

Catinella, B., Schiminovich, D., Cortese, L., et al. 2013, MNRAS, 436, 34

Cen, R., \& Ostriker, J. P. 2006, ApJ, 650, 560

Chabrier, G. 2003, PASP, 115, 763

Cole, S., Lacey, C. G., Baugh, C. M., \& Frenk, C. S. 2000, MNRAS, 319, 168

Conroy, C., \& Wechsler, R. H. 2009, ApJ, 696, 620

D’Agostino, R. B., \& Stephens, M. A. 1986, Goodness-of-Fit Techniques

Dariush, A. A., Raychaudhury, S., Ponman, T. J., et al. 2010, MNRAS, 405, 1873

Davé, R., Cen, R., Ostriker, J. P., et al. 2001, ApJ, 552, 473

Davies, R. D., \& Lewis, B. M. 1973, MNRAS, 165, 231

Duarte, M., \& Mamon, G. A. 2014, MNRAS, 440, 1763

Eckert, K. D., Kannappan, S. J., Stark, D. V., et al. 2015, ApJ, 810, 166

Eckert, K. D., Kannappan, S. J., Stark, D. V., et al. 2016, ApJ, 824, 124

Eke, V. R., Navarro, J. F., \& Frenk, C. S. 1998, ApJ, 503, 569

Feldmeier, J. J., Mihos, J. C., Morrison, H. L., et al. 2004, ApJ, 609, 617

Giodini, S., Pierini, D., Finoguenov, A., et al. 2009, ApJ, 703, 982

Giovanelli, R., Haynes, M. P., Salzer, J. J., et al. 1994, AJ, 107, 2036

Glover, S. C. O., Clark, P. C., Micic, M., \& Molina, F. 2015, MNRAS, 448, 1607

Gonzalez, A. H., Zaritsky, D., \& Zabludoff, A. I. 2007, ApJ, 666, 147

Gonzalez-Perez, V., Lacey, C. G., Baugh, C. M., et al. 2014, MNRAS, 439, 264

Gunn, J. E., \& Gott, J. R., III 1972, ApJ, 176, 1

Guo, Q., Gonzalez-Perez, V., Guo, Q., et al. 2016, MNRAS, 461, 3457

Guo, Q., White, S., Boylan-Kolchin, M., et al. 2011, MNRAS, 413, 101

Gupta, A., Mathur, S., Krongold, Y., Nicastro, F., \& Galeazzi, M. 2012, ApJL, 756, L8

Hambly, N. C., Collins, R. S., Cross, N. J. G., et al. 2008, MNRAS, 384, 637

Haynes, M. P., Giovanelli, R., \& Chincarini, G. L. 1984, ARA\&A, 22, 445

Haynes, M. P., Giovanelli, R., Martin, A. M., et al. 2011, AJ, 142, 170

Hearin, A. P., \& Watson, D. F. 2013, MNRAS, 435, 1313

Hoekstra, H., van Albada, T. S., \& Sancisi, R. 2001, MNRAS, 323, 453

Hou, A., Parker, L. C., Harris, W. E., \& Wilman, D. J. 2009, ApJ, 702, 1199

Jansen, R. A., \& Kannappan, S. J. 2001, Ap\&SS, 276, 1151

Jones, L. R., Ponman, T. J., Horton, A., et al. 2003, MNRAS, 343, 627

Kannappan, S. J. 2004, ApJL, 611, L89

Kannappan, S. J., \& Gawiser, E. 2007, ApJL, 657, L5

Kannappan, S. J., Stark, D. V., Eckert, K. D., et al. 2013, ApJ, 777, 42

Kennicutt, R. C., Jr. 1983a, AJ, 88, 483

Kennicutt, R. C., Jr. 1983b, ApJ, 272, 54

Komatsu, E., Smith, K. M., Dunkley, J., et al. 2011, ApJS, 192, 18

Krick, J. E., Bernstein, R. A., \& Pimbblet, K. A. 2006, AJ, 131, 168

Lacey, C. G., Baugh, C. M., Frenk, C. S., et al. 2016, MNRAS, 462, 3854
Lagos, C. D. P., Baugh, C. M., Lacey, C. G., et al. 2011a, MNRAS, 418, 1649 Lagos, C. d. P., Bayet, E., Baugh, C. M., et al. 2012, MNRAS, 426, 2142

Lagos, C. d. P., Davis, T. A., Lacey, C. G., et al. 2014, MNRAS, 443, 1002

Lagos, C. D. P., Lacey, C. G., Baugh, C. M., Bower, R. G., \& Benson, A. J. 2011b, MNRAS, 416, 1566

Leauthaud, A., George, M. R., Behroozi, P. S., et al. 2012a, ApJ, 746, 95

Leauthaud, A., Tinker, J., Bundy, K., et al. 2012b, ApJ, 744, 159

Leroy, A., Bolatto, A. D., Simon, J. D., \& Blitz, L. 2005, ApJ, 625, 763

Li, C., Kauffmann, G., Fu, J., et al. 2012, MNRAS, 424, 1471

Liang, L., Durier, F., Babul, A., et al. 2016, MNRAS, 456, 4266

Lu, Y., Kereš, D., Katz, N., et al. 2011, MNRAS, 416, 660

Mac Low, M.-M., \& Ferrara, A. 1999, ApJ, 513, 142

Madden, S. C., Cormier, D., \& Remy-Ruyer, A. 2016, in IAU Proc. Vol. 315, From Interstellar Clouds to Star-Forming Galaxies: Universal Processes? (Cambridge: Cambridge Univ. Press), 191

Main, R., McNamara, B., Nulsen, P., Russell, H., \& Vantyghem, A. 2016, MNRAS, 464, 4360

Melioli, C., Brighenti, F., \& D’Ercole, A. 2015, MNRAS, 446, 299

Merson, A. I., Baugh, C. M., Gonzalez-Perez, V., et al. 2016, MNRAS, 456, 1681

Mitchell, P. D., Lacey, C. G., Baugh, C. M., \& Cole, S. 2016, MNRAS, 456, 1459

Mitchell, R. J., Ives, J. C., \& Culhane, J. L. 1977, MNRAS, 181, 25P

Moffett, A. J., Kannappan, S. J., Berlind, A. A., et al. 2015, ApJ, 812, 89

Morrissey, P., Conrow, T., Barlow, T. A., et al. 2007, ApJS, 173, 682

Moster, B. P., Naab, T., \& White, S. D. M. 2013, MNRAS, 428, 3121

Moster, B. P., Somerville, R. S., Maulbetsch, C., et al. 2010, ApJ, 710, 903

Pfenniger, D., \& Revaz, Y. 2005, A\&A, 431, 511

Pineda, J. L., Langer, W. D., Velusamy, T., \& Goldsmith, P. F. 2013, A\&A, 554, A103

Planck Collaboration, Ade, P. A. R., Aghanim, N., et al. 2014, A\&A, 571, A16

Ponman, T. J., Allan, D. J., Jones, L. R., et al. 1994, Natur, 369, 462

Ramella, M., Boschin, W., Geller, M. J., Mahdavi, A., \& Rines, K. 2004, AJ, 128, 2022

Revaz, Y., Pfenniger, D., Combes, F., \& Bournaud, F. 2009, A\&A, 501, 171

Roberts, I. D., Parker, L. C., \& Karunakaran, A. 2016, MNRAS, 455, 3628

Robotham, A. S. G., Norberg, P., Driver, S. P., et al. 2011, MNRAS, 416, 2640

Röllig, M., Ossenkopf, V., Jeyakumar, S., Stutzki, J., \& Sternberg, A. 2006, A\&A, 451, 917

Schruba, A., Leroy, A. K., Walter, F., et al. 2012, AJ, 143, 138

Skrutskie, M. F., Cutri, R. M., Stiening, R., et al. 2006, AJ, 131, 1163

Smith, B. D., Hallman, E. J., Shull, J. M., \& O’Shea, B. W. 2011, ApJ, 731, 6

Sokołowska, A., Mayer, L., Babul, A., Madau, P., \& Shen, S. 2016, ApJ, 819,21

Springel, V., White, S. D. M., Jenkins, A., et al. 2005, Natur, 435, 629

Stark, D. V., Kannappan, S. J., Eckert, K. D., et al. 2016, ApJ, 832, 126

Stoughton, C., Lupton, R. H., Bernardi, M., et al. 2002, AJ, 123, 485

Strauss, M. A., Weinberg, D. H., Lupton, R. H., et al. 2002, AJ, 124, 1810

Swaters, R. A., Sancisi, R., van der Hulst, J. M., \& van Albada, T. S. 2012, MNRAS, 425, 2299

Taylor, C. L., Kobulnicky, H. A., \& Skillman, E. D. 1998, AJ, 116, 2746

Tully, R. B., Rizzi, L., Dolphin, A. E., et al. 2006, AJ, 132, 729

von Benda-Beckmann, A. M., D’Onghia, E., Gottlöber, S., et al. 2008, MNRAS, 386, 2345

Wang, L., Dutton, A. A., Stinson, G. S., et al. 2017, MNRAS, 466, 4858

Warren, M. S., Abazajian, K., Holz, D. E., \& Teodoro, L. 2006, ApJ, 646, 881

Weinmann, S. M., van den Bosch, F. C., Yang, X., \& Mo, H. J. 2006, MNRAS, 366, 2

White, S. D. M., Navarro, J. F., Evrard, A. E., \& Frenk, C. S. 1993, Natur, 366, 429

Wolfire, M. G., Hollenbach, D., \& McKee, C. F. 2010, ApJ, 716, 1191

Yang, X., Mo, H. J., van den Bosch, F. C., et al. 2007, ApJ, 671, 153

Yang, X., Mo, H. J., \& van den Bosch, F. C. 2009, ApJ, 695, 900 